\title{
Feeding properties and behavior of hammer- and knife-milled pine
}

Tyler L. Westover, Sergio Hernandez, Austin C. Matthews, J. Chadron Benjamin Ryan, C. Luke Williams

October 2017

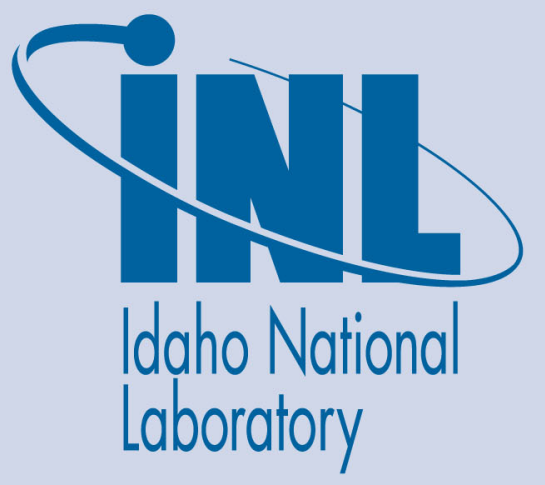

The INL is a U.S. Department of Energy National Laboratory operated by Battelle Energy Alliance 


\title{
Feeding properties and behavior of hammer- and knife-milled pine
}

\author{
Tyler L. Westover, Sergio Hernandez, Austin C. Matthews, J. Chadron Benjamin \\ Ryan, C. Luke Williams
}

October 2017

Idaho National Laboratory Idaho Falls, Idaho 83415

http://www.inl.gov

Prepared for the

U.S. Department of Energy

Office of Energy Efficiency and Renewable Energy

Under DOE Idaho Operations Office

Contract DE-AC07-05ID14517 


\section{Feeding properties and behavior of hammer- and knife-milled pine}

Sergio Hernandez, Tyler L. Westover*, Austin C. Matthews, J. Chadron B. Ryan, C. Luke Williams

Idaho National Laboratory, 2525 Fremont Ave., Idaho Falls, ID 83415, USA

* Corresponding author. Tel. 208-526-1553. E-mail address: tyler.westover@inl.gov (T. L.

Westover)

\section{Key Words}

Powder flow function; Effective angle of internal friction; Schulze ring shear cell; Particle distribution; Moisture content

\section{Introduction}

Considerable research in utilizing lignocellulosic biomass as a feedstock for renewable fuels and chemicals has been driven by energy and environmental concerns over petroleum consumption $[1,2]$. However, using biomass as a renewable resource is challenging because raw biomass feedstocks have great variability in their physical and chemical properties, and this variability has unfavorable effects on biofuels conversion processes [3]. While the effect of biomass heterogeneity on conversion processes is something to be considered, there are also substantial problems in consistently supplying biomass to the actual conversion system. A study by Merrow of 500 projects found a strong correlation between the level of difficulty of material handling and the time required for plant start up, with plants that handle raw bulk solids requiring more than two times longer to start up than plants handling gases or liquids [4]. A survey specific to the biofuels industry was conducted in 2014 to gauge biomass feeding and handling difficulties. All of the approximately 20 responses from different companies reported multiple substantial difficulties in feeding different types of biomass [5]. To prevent such problems, the flowability characteristics of biomass feedstocks must be understood to reduce or prevent feeding problems in biorefineries [6,7].

Biomass material flow properties can be characterized as irregular due to cohesion or interparticle mechanical interference causing problems in feeding behavior [8]. Additionally, particle shape influences arching over openings. For instance, long or thin biomass particles can increase arching tendencies, along with increasing moisture levels that often heighten arching [9,10,11]. Other factors that contribute to arching and decreased flowability are the depth of hopper opening, particle size distribution and low material densities [6,7].

This variability in biomass properties affects not only the specific conversion process but also the supply of biomass to the conversion systems. Kenny et al. described that the handling and feeding system performance of lignocellulosic biomass feedstocks is determined by many factors including: particle size and shape distributions, bulk density, chemical composition of particles, moisture content, temperature, presence of trapped gases and the unique stress (i.e., compaction) history of the material [12]. The interdependency of these flow-related physical properties makes the study and operation of feed handling systems even more complex [13]. For instance, the shape of lignocellulosic biomass is extremely irregular due to the variable content of cellulose, hemicellulose and lignin and generally must also be considered in feeding and conversion processes [14]. 
Currently, the data required to solve biomass handling problems are scarce and much more information on flow properties of lignocellulosic biomass is needed to reduce the risk of process shutdowns in biorefineries and improve the cost-competitiveness of biofuel production $[7,11,12,15,16]$. However, conventional methods may not be suitable to characterize and predict the flow behavior of biomass materials. For example, the high compressibility, elasticity, and irregular shapes of biomass materials can require very long strain displacement before shear failure and the attainment of steady state shear stress $[7,17,18]$. Another source of error is that the conventional design procedures of feeding and handling equipment were developed for powders made of rigid and non-fibrous particles that follow the Mohr-Coulomb model [17,19]. A variety of shear testing methods have been devised and evaluated for a wide range of materials as summarized by Schwedes [20], and other methods have also been developed and applied for specific applications [10,21]; however, a systematic approach that is demonstrated to adequately characterize and understand the flow behavior of compressible, cohesive, and anisotropic materials has yet to emerge.

In this work, the feeding performance of a range of clean hammer- and knife-milled lodgepole pine samples with varying moisture contents and grind sizes are evaluated in a custom wedgeshaped hopper and a screw feeder equipped with three different screw configurations. Results from feeding tests were compared to measured rheological properties of the samples, including bulk density, compressibility, elastic recovery cohesive strength/unconfined yield strength, and angle of internal friction. These properties are used to predict the minimum hopper opening that is required to ensure reliable flow, and these predictions are compared to values measured using the custom wedge-shaped hopper. The comparison between experiments and predictions offers insights into the suitability of current methods to understand the feeding behavior of biomass materials as well as future work that is needed to improve the state of technology.

\section{Materials and methods}

\subsection{Materials}

Chipped lodgepole pine (pinus contorta) was obtained from Tricon Timber Mill located in Mineral County, Montana and was ground using 1.6, 3.0, 13 and $25 \mathrm{~mm}$ screens in a SchutteBuffalo hammer mill (Circ-U-Flow model 18-7-300) and a Thomas Model 4 Wiley knife mill (Thomas Scientific, model no. 3375-E55) equipped with a $6.0 \mathrm{~mm}$ screen. A riffle sample splitter with $25 \mathrm{~mm}$ wide riffles was used to split small quantities of samples while larger samples quantities were divided using a custom rotary splitter that consists of a conveyor and eight bins mounted on a rotating table. The $45 \mathrm{~L}$ bins rotated over 100 times during each splitting operation to ensure the samples were representative of the original sample. The different pine grinds were prepared at moisture contents (MCs) ranging from $10 \%$ to $40 \%$ as shown in Table 1 to form a set of 17 unique samples. For comparison, loblolly pine pellets were obtained from the Biomass Feedstocks National User Facility (BFNUF) at INL and included in the test matrix. Moisture was added to the milled samples by spraying the desired quantity of water on the samples in large plastic bags, sealing the bags, and then mixing the contents thoroughly before storing at approximately $5{ }^{\circ} \mathrm{C}$ for a minimum of 48 hours to allow the moisture to be absorbed homogeneously by the wood. 
Table 1. Grind sizes and moisture contents of tested samples (18 total)

\begin{tabular}{|c|c|c|c|c|}
\hline Pine grind size & \multicolumn{5}{|c|}{ Moisture contents (\%) } \\
\hline $25 \mathrm{~mm}$ & 10 & 20 & 30 & 40 \\
\hline $13 \mathrm{~mm}$ & 10 & 20 & 30 & 40 \\
\hline $6 \mathrm{~mm} *$ & 10 & 20 & 30 & - \\
\hline $3 \mathrm{~mm}$ & 10 & 20 & 30 & - \\
\hline $1.6 \mathrm{~mm}$ & 10 & 20 & 30 & - \\
\hline Pellet & 8 & - & - & - \\
\hline
\end{tabular}

* Prepared using a knife mill

Samples with particle sizes smaller than $13 \mathrm{~mm}$ were prepared with a maximum of $30 \% \mathrm{MC}$ because that is the highest MC that is typically observed after fine grinding. Moisture content was measured following American Society of Agricultural and Biological Engineers (ASABE) S358.2. Briefly, this procedure involved placing a 50-100 $\mathrm{g}$ of sample in a horizontal convective oven at an approximate temperature of $105^{\circ} \mathrm{C}$ for a period of $24-30 \mathrm{~h}$. The mass loss of the samples was assumed to be primarily moisture and was reported on a wet basis.

\subsection{Sieve particle classification}

Sieve classification of the materials was performed in duplicate according to ASAE S319.3 using a standard Ro-Tap separator (W.S. Tyler model RX-29). U.S.A. standard test sieves with sizes of $15.0,9.5,6.3,4.75,3.35,2.0,1.7,1.18,0.425,0.3,0.25,0.18$, and $0 \mathrm{~mm}$ (pan), although not all sieves were employed for each grind size. The cumulative particle passing distributions (CPDs) and the associated probability density distributions (PDDs), which represent the derivative of the CPDs, were calculated. For all of the analyses, the 50\% cumulative passing percentile sieve size, Sieve 50, was calculated by interpolation to find the theoretical sieve size that corresponds to retaining $50 \%$ of the particles by mass. Similarly, the $10 \%$ and $90 \%$ cumulative passing percentile sieve sizes (Sieve10 and Sieve90, respectively) were also calculated and reported.

\subsection{Coarse particle image analysis (Clemex automated digital image analysis)}

The size and shape properties of the particles in each grind size were determined using a Clemex digital image analysis system (Clemex Technologies Inc., QC, Canada) at 48X magnification. The method used is described in detail by Westover et al. [18,22]. Digital image analysis was performed by sparsely sprinkling representative samples onto a black moving conveyor belt, while images were captured by a camera (Clemex L 1.4 C CCD: 1392 x 1024 pixels) positioned approximately 1 meter above the particles. Particles were separated from the background using a grey threshold. Additional tests demonstrated that results were not affected by particle orientation or conveyor belt speed. Each set of images was analyzed cumulatively to determine particle area, perimeter, length, width, aspect ratio (AR), and roughness (Rough.). The software also allows particles with certain roughness values (ratio of convex perimeter to actual perimeter) to be excluded from the measurement statistics, and this feature was employed to prevent overlapping particles from skewing the measurements. Particles extending outside the digital image were also excluded from analysis. Finally, particles with widths less than 5 camera pixels (approximately $0.4 \mathrm{~mm}$ ) were also discarded from the analysis due to a lack of resolution.

Similar to the sieve analysis, CPDs and PDDs were determined for primary particle parameters measured by the digital imaging method and were used to estimate $10 \%, 50 \%$, and $90 \%$ cumulative passing values. The tests for each sample material were performed separately three times in order to estimate repeatability. An average of more than 20,000 particles were analyzed 
per sample. Calibration of the digital imaging system and assessment of performance for analyzing pine particles has been published in a prior publication [18].

\subsection{Bulk density, Compression and Elastic Recovery Tests}

The bulk densities of the samples were measured by following a modified version of ASTM E873-82 for densified particulate biomass fuels [23]. The samples were poured into a cylindrical container from a height of $0.6 \mathrm{~m}$ above the container's top edge until the height of the material was approximately $66 \%$ of the container's diameter. The height of the material was less than that normally used in the ASTM E 873-82 in order to minimize the effects of the walls on subsequent compression tests. The loose bulk density of the sample was estimated by dividing the average sample height, as determined by five or more measurements at separate locations, by the mass of the sample. To promote settling, the container with the sample was then dropped five times from a height of $0.15 \mathrm{~m}$ onto a hard surface. Subsequently, the 'tapped bulk density' was determined following the density measurement procedure described above.

After the specimens were packed by dropping from a fixed height, the container was loaded into a universal load frame (Instron 5982 Dual Column Floor Frame) that had a cylindrical lid attached to its crosshead. The diameter of the lid was slightly smaller than that of the container to minimize potential friction effects between the lid and the container. The Instron universal testing machine was then used to slowly lower the cylindrical lid to compress the sample to 10 $\mathrm{kPa}$ of uniaxial pressure and maintain that pressure for 10 minutes while simultaneously measuring the sample volume. After 10 minutes, the pressure was reduced to $0.07 \mathrm{kPa}$ for 10 minutes to allow the material to elastically recover against a near zero pressure. The bulk density tests were repeated a minimum of six times, while the compressibility and elastic recovery tests were repeated a minimum of two times.

\subsection{Hopper arching Tests}

A custom hopper, with an adjustable outlet, was used to study the arching tendency of lodgepole pine subjected to different comminution methods (hammer mill vs. knife mill) and at multiple moisture contents. The unique feature of this custom hopper is that the size of the outlet can be continuously altered while maintaining symmetry and without disturbing the material through friction forces due to a moving gate. The details of the hopper and its operation have been described in a previous open-access publication [18]. Briefly, as shown in Appendix A Fig. A.2 the hopper is $\mathrm{V}$-shaped (wedge-shaped) and designed to allow the sloping walls to be raised, which effectively increases the size of the outlet at the bottom. A stationary liner made of 0.1 $\mathrm{mm}$ stainless steel foil is inserted between the sample and the inclined walls, so that even though the walls slide upwards, the material inside the hopper does not encounter dynamic friction because of the stationary liner. If desired, material loaded into the hopper can be packed in layers and weights can be applied to the top surface of the material to subject the material to compressive pressure. A typical test that includes weights is conducted by filling the hopper in layers of approximately $8 \mathrm{~cm}$ and applying $3 \mathrm{kPa}$ briefly to each layer. This process is repeated until a material depth of approximately $37 \mathrm{~cm}$ is attained, and then $3 \mathrm{kPa}$ of pressure is applied to the top surface of the material. The biomass sample is allowed to settle for 5 minutes while subjected to pressure, and then the walls are slowly raised to increase the size of the hopper outlet until the arch of material in the bottom of the hopper fails and material flows. The final width of the hopper opening is recorded as the critical arching width, $W_{\text {min,meas. }}$ Each 
measurement is repeated a minimum of three times in order to assess measurement variability. For all tests reported here, the hopper outlet was fully closed at the beginning of the tests.

\subsection{Auger Feeder Tests}

Auger feeding tests were conducted using an Acrison Bin Weight Screw Feeder (model 402X250-75-BDF1.5-E/2) equipped with either a small or a large screw auger with diameters of 45 $\mathrm{mm}$ and $63 \mathrm{~mm}$, respectively. To promote consistent feeding, the bin is equipped with two conditioning agitators that operate on either side of the screw auger as shown in Appendix A Fig. A.6. The screw augers have constant pitch and constant diameter, which causes material to be preferentially removed from the front of the hopper. An Acrison SBC-2000 DSP feeder controller with set calibration values of scale, feeder and material variations were used for the feeding tests. Factory-set values of proportional, integral, and derivative (PID) settings of 5.0, 5.0 seconds, and 0, respectively, as recommended by the vendor were used in feeder tests. Volumetric feed tests were performed first at $20 \%$ and $40 \%$ of maximum auger speed (volumetric feeding mode) and were labeled Vol-slow and Vol-fast, respectively. After the volumetric flow tests were completed, gravimetric tests were performed to match the volumetric feed rates (in $\mathrm{kg} / \mathrm{hr}$ ). These tests were labeled Grav-slow and Grav-fast respectively. For each test, material was fed out of the auger using constant auger feed settings. The mass feed rate was recorded by the feeder controller at $5 \mathrm{~Hz}$ and archived in a laptop computer. Material exiting the auger was captured in a container on a Mettler-Toledo (model XS6002S) scale that recorded the mass accumulation at 1 second intervals. The real-time power consumption of the auger feeder was also recorded at $5 \mathrm{~Hz}$ frequency using a power meter. After the tests, feeding data was analyzed to determine the average feed rate $Q(\mathrm{~kg} / \mathrm{hr})$ and also the time variability of the feed rate $S_{Q}$, which was calculated as the standard deviation of the feed rate for a period of at least 2 minutes after the feed rate had stabilized. For all tests, the bin supplying material to the feeding auger was filled to approximately $25 \%$ of maximum capacity. All tests were repeated a minimum of two times.

\subsection{Shear Tests and Hopper Flow Prediction Calculations}

Shear strength measurements were performed using an automated Schulze ring shear tester (Dietmar Schulze Schüttgutmesstechnik, Wolfenbüttel, Germany) in accordance with ASTM D6773-08 using a size M shear cell (outer diameter of $20 \mathrm{~cm}$ and inner diameter of $10 \mathrm{~cm}$ ). The shear strength test method is also described in a previous publication [18]. Following the method of Jenike [19], the shear tests were used to calculate the minimum hopper outlet width that ensures consistent flow $W_{\text {min,pred. }}$ Briefly, shear tests were conducted to measure the instantaneous yield loci (IYL) of the materials for preshear compression stresses of $0.5 \mathrm{kPa}, 2.0$ $\mathrm{kPa}$ and $7 \mathrm{kPa}$. Shear tests conducted using pine pellets with a preshear stress of $0.5 \mathrm{kPa}$ did not produce reliable results; consequently, additional tests were performed using pellets and a few other materials with a preshear stress of $1.0 \mathrm{kPa}$. The preshear stresses listed above were chosen based upon recommendations for silo design in which it has been observed that consolidation stress generally increases with material bulk density and equipment scale and ranges from 4 to 8 $\mathrm{kPa}$ for many applications [24]. For bulk densities between 150 and $300 \mathrm{~kg} / \mathrm{m}^{3}$ (such as the pine materials used in this work), expected consolidation stresses range from approximately $1.5 \mathrm{kPa}$ for $100 \mathrm{~L}$ containers (such as the custom hopper used in this work) to $7 \mathrm{kPa}$ for medium and large silos [24]. A minimum preshear stress of $0.5 \mathrm{kPa}$ was selected for measuring the IYL because that preshear stress requires a minimum applied normal stress of approximately $0.1 \mathrm{kPa}$ 
(20\% of the preshear stress) [24], which is the minimum normal load that is recommended for tests using the Schulze ring shear tester [25]. This recommendation is reasonable because $0.1 \mathrm{kPa}$ represents $0.5 \%$ of the rated normal load that the Schulze shear tester can apply. As shown in the results section below, these equipment limitations cause large uncertainties in the shear flow properties of the pine samples featured in this work because the IYL must be extrapolated to low normal stresses, and even small inaccuracies in measurements of shear points can have a large influence on the magnitude of measured shear properties [24,26].

A minimum of four points were measured on each IYL, with points corresponding to the smallest pressure being tested first and then repeated at the end of the test to verify that the material strength had not changed more than 5\% during the course of the test as recommended in the equipment user manual [24]. Many of the IYL measurements were repeated three or more times, so that a minimum of five separate shear tests that included a total of 20 individual shear measurements were performed for each material. The IYL from the shear tests were analyzed as described in $[18,26,27]$ to determine the major principal compressive stress $\left(\sigma_{1}\right)$, the effective angle of internal friction $\delta_{\text {eff }}$, and the unconfined yield stress $\left(\sigma_{c}\right)$ (also called the unconfined yield strength and denoted $f_{c}$ ) for each test. Jenike's method [19] using the flow function approach was then used to predict the minimum hopper outlet width needed to ensure consistent flow. Wall friction tests were also conducted using the wall friction cell as specified in the user documentation [24].

Material flow functions, MFFs, are curves that plot $f_{c}$ as a function of $\sigma_{1}$ and represent the stress needed to collapse an arch as a function of the stress that caused the arch to form. The shear stress required to collapse an arch is often referred to as the critical applied shear stress $\tau_{c r i t}$. The relationship between $\tau_{c r i t}$ and theoretical minimum hopper outlet width that ensures consistent flow $W_{\text {min,pred }}$ (i.e. the critical arching width) is given by $[19,28]$

$$
W_{\text {min,pred }}=H(\theta) \frac{\tau_{\text {crit }}}{\rho_{b} g}
$$

where $\theta$ is the hopper semi-angle (the angle of the hopper wall with respect to the vertical axis), $H(\theta)$ is a function equal to $1+\theta / 180$ for wedge-shaped hoppers, $\rho_{\mathrm{b}}$ is the material bulk density, and $g$ is the acceleration of gravity. For these tests, $\theta=32^{\circ}$, so $H(\theta)=1.18$.

The critical applied stress $\tau_{\text {crit }}$ lies on the material flow function, MFF, and is found by determining the major principle stress, $\sigma_{\text {crit }}$. that caused the arch to form. The assumptions used to determine $\sigma_{\text {crit }}$ depened upon the specific flow conditions. Here we use two approaches. In the first approach, as kindly suggested by a peer-reviewer, we assume that $\sigma_{\text {crit }}$ is equal to the hydrostatic pressure caused by the material above the arch and also any applied weight. The depth of material above the arch was determined after the test by assuming that the arch was located at the bottom of the hopper walls after the arch failed. The mathematical formula is $\sigma_{c r i t}$ $=\rho \mathrm{b} g\left(h_{\text {bed }}-0.5 W_{\text {min,meas }} \tan (\theta)+\sigma_{\text {weigth }}\right.$, where $h_{\text {bed }}$ equals the depth of material in the hopper (37 $\mathrm{cm}$ for most tests) and $\sigma_{\text {weight }}$ equals the pressue due to any applied weights (either 0 or $3 \mathrm{kPa}$ ). It may be observed that this formula for $\sigma_{\text {crit }}$ actually estimates the vertical component of the compressive stress, not the major principal stress, which is likely somewhat higher, so that this method provides a lower limit for $\sigma_{\text {crit. }}$ 
The second method to estimate $\sigma_{\text {crit }}$ is based upon Jenike's flow function approach. Using solid mechanics, Jenike showed that in a mass flow hopper, the ratio of the compacting stress to the shear stress is uniform. He called this term the hopper flow factor ff [19] and published charts from which $f f$ can be determined as a function of $\delta$ eff, the kinematic angle of friction between the hopper wall and the flow material, and the hopper semi-angle $\theta$ [19]. The point at which the inverse hopper flow factor (1/ff) intercepts the material flow function, MFF, marks the stress condition at which the arch abutment stresses exceed the strength of material in the arch, causing the arch to fail. This point can be determined graphically or mathematically (because the hopper flow factors $f f$ are ratios of $\sigma_{1}$ to $f_{c}$, they can be plotted in the same diagram as the material flow functions). Jenike showed that the compacting stress decreases as material moves downward through the hopper, so that this approach provides an estimate for the upper limit of the stress that caused the arch to form. Based upon the discussion above, the two methods of estimating $\sigma_{c r i t}$ should provide lower and upper limits for estimating $W_{\text {min,pred. }}$

\section{Results and Discussion}

\subsection{Evaluation of sieve particle classification and coarse particle image analysis}

Particle cumulative passing distributions (CPDs) based upon the width and sieve analyses are presented in Fig. 1. Corresponding CPDs for particle length are shown in Appendix A Fig. A.1. Statistics from the particle size and shape distributions from the image and sieve analyses are summarized in Table 2. As noted above, particles with widths less than approximately $0.4 \mathrm{~mm}$ were excluded from the digital imaging analyses because of limited camera resolution. Sieve analysis indicated that particles with diameters less than $0.4 \mathrm{~mm}$ represented less than $1 \%$ of the total mass of the pellets and less than approximately $1,2,5,13$, and $22 \%$ of the total mass of the $25,13,6^{*}, 3$, and $1.6 \mathrm{~mm}$ grind materials, respectively. Table 2 also shows the geometric mean particle sizes, which were calculated as the square root of the product of Width and Length as measured by the digital camera.

In the analysis of the woody samples, the camera image method reports slightly larger particle widths for the $13 \mathrm{~mm}$ and $25 \mathrm{~mm}$ grinds than the sieve method. This is likely because the image method uses the shortest of 32 Feret diameters measured approximately every $5.6^{\circ}$ for each object, so the image method can slightly overestimate particle width for particles that are not well aligned with the Feret measurements. The difference between the camera image and the sieve methods is greater for smaller particles (less than approximately $2 \mathrm{~mm}$ ), which is likely due to greater error associated with image resolution and color threshold smoothing between the particles and the black background conveyor belt. Another consideration is that sieve analysis tends to separate particles by length as well as by diameter, although the relatively low aspect ratios of the wood chips in this study (approximately 2.6 for the $3 \mathrm{~mm}$ and $6^{*} \mathrm{~mm}$ grinds and 3.8 for the $13 \mathrm{~mm}$ grind) indicates that sieving analysis is likely dominated by particle diameter not length. One item to note is that the particle size/shape distribution characteristics of the $6 \mathrm{~mm}$ knife-milled materials are generally similar to those of the $3 \mathrm{~mm}$ hammer-milled materials, except for the measured sieve widths, which are somewhat larger. 


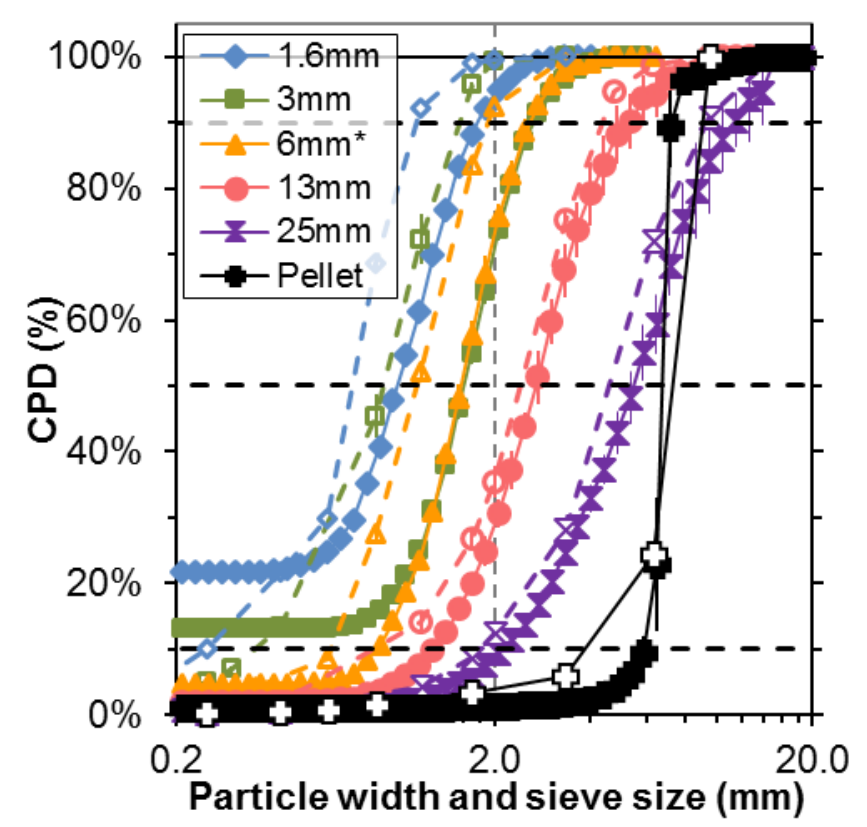

Figure 1. Particle width cumulative passing distributions (CPDs) for ground pine samples determined by the camera imaging (solid symbols) and sieve analysis (hollow symbols). Repeatability bars, which are often smaller than the symbols, indicate the standard deviation of multiple replicates. The camera CPDs incorporate offsets to account for particles with widths less than $0.4 \mathrm{~mm}$, which were removed prior to analysis.

Table 2. Particle size and shape distribution statistics from the image and sieve analyses. For digital imaging analyses, values in parenthesis indicate the standard deviation of three replicates; for sieve analyses values in parenthesis indicate the difference between duplicate analyses. N/A indicates parameters that were not calculated due to limited camera resolution.

\begin{tabular}{|c|c|c|c|c|c|c|}
\hline & \multicolumn{3}{|c|}{ Sieve (mm) } & \multicolumn{3}{|c|}{ Width $(\mathrm{mm})$} \\
\hline Sample & Sieve50 & Sieve 10 & Sieve90 & $W_{50}$ & $W_{10}$ & $W_{90}$ \\
\hline Pellet & $7.39(0.03)$ & $4.02(0.07)$ & $9.08(0.01)$ & $6.73(0.08)$ & $5.94(0.22)$ & $7.29(0.21)$ \\
\hline $25 \mathrm{~mm}$ & $4.82(0.06)$ & $1.80(0.01)$ & $9.36(0.07)$ & $5.48(0.34)$ & $2.11(0.07)$ & $11.6(2.23)$ \\
\hline $13 \mathrm{~mm}$ & $2.5(0.02)$ & $0.92(0.01)$ & $4.42(0.02)$ & $2.70(0.10)$ & $1.29(0.04)$ & $5.25(0.64)$ \\
\hline $6 \mathrm{~mm}^{*}$ & $1.15(0.08)$ & $0.62(0.05)$ & $1.92(0.04)$ & $1.58(0.04)$ & $0.85(0.00)$ & $2.57(0.1)$ \\
\hline $3 \mathrm{~mm}$ & $0.91(0.06)$ & $0.36(0.01)$ & $1.57(0.07)$ & $1.61(0.02)$ & N/A & $2.65(0.06)$ \\
\hline $1.6 \mathrm{~mm}$ & $0.73(0.04)$ & $0.25(0.02)$ & $1.15(0.03)$ & $0.99(0.02)$ & N/A & $1.77(0.06)$ \\
\hline
\end{tabular}

\begin{tabular}{|c|c|c|c|c|c|c|}
\cline { 2 - 7 } \multicolumn{1}{c|}{} & \multicolumn{3}{c|}{ Length (mm) } & AR (-) & Rough. (-) & GM (mm) \\
\cline { 3 - 7 } Sample & L50 & $L_{10}$ & $L_{90}$ & AR50 & Rough50 & $G M$ \\
\cline { 2 - 7 } Pellet & $10.8(0.3)$ & $7.5(0.3)$ & $15.1(0.5)$ & $1.60(0.00)$ & $0.86(0.01)$ & 8.5 \\
$25 \mathrm{~mm}$ & $17.9(0.5)$ & $7.6(0.3)$ & $30.1(0.9)$ & $3.04(0.26)$ & $0.94(0.00)$ & 9.9 \\
$13 \mathrm{~mm}$ & $10.7(0.3)$ & $4.4(0.1)$ & $18.9(0.7)$ & $3.77(0.05)$ & $0.96(0.00)$ & 5.2 \\
$6 \mathrm{~mm} *$ & $4.0(0.1)$ & $2.1(0.0)$ & $7.2(0.4)$ & $2.51(0.03)$ & $1.00(0.00)$ & 2.5 \\
$3 \mathrm{~mm}$ & $4.3(0)$ & N/A & $6.7(0)$ & $2.57(0.02)$ & $1.00(0.00)$ & 2.6 \\
$1.6 \mathrm{~mm}$ & $2.9(0)$ & N/A & $5(0)$ & $2.84(0.02)$ & $1.00(0.00)$ & 1.7 \\
\hline
\end{tabular}




\subsection{Evaluation of bulk density, compression and elastic recovery tests}

Figure 2A displays tapped bulk densities for ground pine. As expected, the bulk density increases with moisture content; however, surprisingly there is not a clear trend of bulk density with particle size. The smallest particle size $(1.6 \mathrm{~mm}$ grind $)$ has the highest bulk density for its respective moisture content, while the $3 \mathrm{~mm}$ and $13 \mathrm{~mm}$ grinds have the lowest bulk densities. Uncertainty bars in the figure show the standard deviations of the tests. Figures $2 \mathrm{~B}$ and $2 \mathrm{C}$ show the measured compression and elastic recovery (springback) ratios. The compression ratio is defined as the ratio of bulk density with $10 \mathrm{kPa}$ applied pressure to the tapped bulk density. The milled materials exhibit compression ratios of 1.1 to 1.37 , with the $3 \mathrm{~mm}$ and 25 materials having the highest and lowest compressibilities, respectively. As moisture content increased, all of the materials became more compressible. The elastic recovery is the ratio of measured bulk volumes after and during application of $10 \mathrm{kPa}$ pressure. After the load was released, the materials expanded less than $10 \%$, with the $30 \%$ and $40 \%$ moisture content materials expanding the most. The bulk density of the pellets was 4-5 times that of the ground samples, and the pellets had much lower compressibility and elastic recovery.

In general, materials that are more compressible and have greater elastic recovery exhibit more severe feeding and handling problems. For example, in wedge or conical shaped hoppers, as material descends through the narrowing portion of the hopper, the walls carry more of the weight of material overhead, such that the compressive pressure decreases toward the hopper outlet, where it essentially becomes zero when the gate is open (the hopper opening is a free surface). For incompressible materials, the decreasing compressive force as material moves down the hopper reduces interparticle friction forces to make the material more flowable at the hopper outlet. However, highly compressible materials can expand in the hopper nozzle, which maintains the compressive pressure and material strength, and potentially causes material to become wedged above the hopper gate [21].

\subsection{Evaluation of arching Tests}

Figure 3 displays the measured minimum outlet widths for experiments with and without $3 \mathrm{kPa}$ of applied pressure. The 1.6 hammer-milled and $6 \mathrm{~mm}$ knife-milled samples tended to exhibit substantially better flow behavior than the $3 \mathrm{~mm}$ and $13 \mathrm{~mm}$ hammer-milled samples. The fact that the $6 \mathrm{~mm}$ knife-ground samples flowed better than the $3 \mathrm{~mm}$ and $13 \mathrm{~mm}$ hammer-ground samples is likely due to differences in microscopic surface roughness from the different grinding operations and not from macroscopic particle shape or roughness characteristics reported in Table 2. For example, as shown in Table 2, the aspect ratios of the $6 \mathrm{~mm}$ samples are similar to those of the $3 \mathrm{~mm}$ samples, but all other measured size and shape properties of the $6 \mathrm{~mm}$ sample are intermediate between those of the $3 \mathrm{~mm}$ and $13 \mathrm{~mm}$ samples. Related research has previously shown that knifed-chopped switchgrass has substantially better flow behavior than hammerground switchgrass at low consolidation pressures [18], similar to what is observed for pine in this work. At higher consolidation pressures, however, the flow differences between knifechopped and hammer-milled switchgrass decrease, as also shown for pine in Fig. 3B.

For the unweighted tests, only the $25 \mathrm{~mm}$ grind exhibited a strong effect of $\mathrm{MC}$ with $W_{\text {min,exp }}$ increasing with increasing moisture content up to $30 \% \mathrm{MC}$. For the tests with $3 \mathrm{kPa}$ applied pressure, the $10 \% \mathrm{MC}$ samples consistently flowed through narrower openings than samples with higher moisture contents, except for the $6 \mathrm{~mm}$ knife-milled sample. The $25 \mathrm{~mm}$ grind 

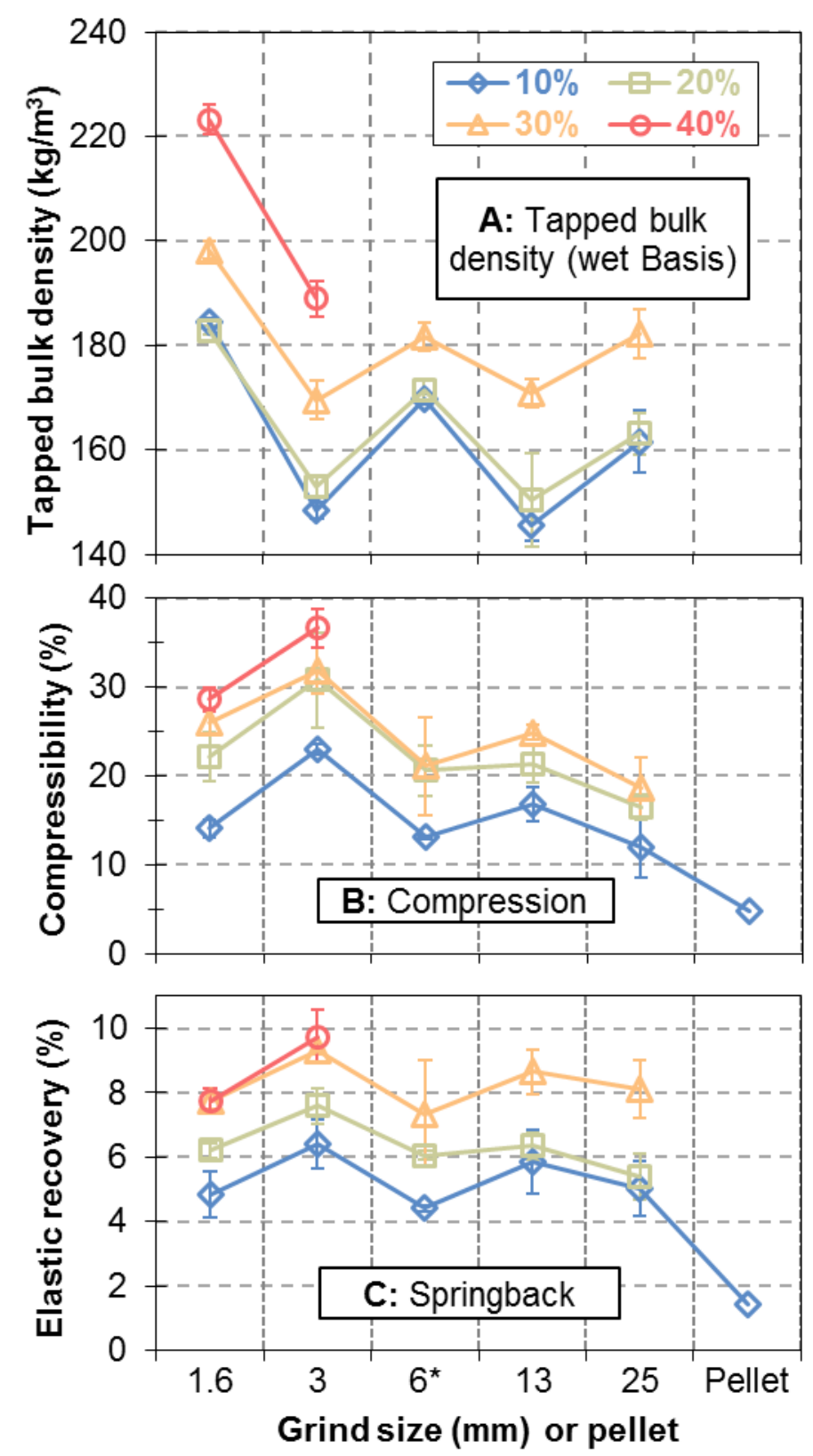

Figure 2. Bulk density measurements of the pine materials. Panels A, B and C show tapped bulk density on a wet basis, compression ratio and springback ratio, respectively. The bulk density of the pellets (not shown) was $805 \mathrm{~kg} / \mathrm{m}^{3}$ with a standard deviation of $20 \mathrm{~kg} / \mathrm{m}^{3}$.

sample flowed through a much narrower hopper outlet than any of the other samples, except for the pellets. Table 2 indicates that the geometric mean particle size of the pellets and the $25 \mathrm{~mm}$ grind was $0.85 \mathrm{~mm}$ and $0.99 \mathrm{~mm}$, respectively. Comparing these values to the measured outlet sizes $W_{\text {min,meas }}$ for the flow of these materials, shows that the ratio of the geometric mean particle size to $W_{\text {min,meas }}$ was approximately 4 and 9 for the pellets and $2 \mathrm{~mm}$ grind, respectively. This ratio was 20 or greater for all of the other milled samples. Interlocking of particles through narrow openings is usually important when the size of the opening is less than approximately 10 times the geometric mean particle size, so interlocking of particles is expected to be important for the flow of the pellets and for the unweighted $25 \mathrm{~mm}$ grinds with low moisture contents. We 
also note that moisture can have competing effects on flow behavior. For example, it can increase particle cohesion through surface tension or or act as a lubricant to increase flow. High levels of moisture also increases material bulk density to increase gravity flow. Adding $3 \mathrm{kPa}$ of pressure (Figure 3B) significantly increased the measured critical arching width, $W_{\text {min,meas }}$, for all samples, except the pellets. The tests that employed $3 \mathrm{kPa}$ of applied pressure are intended to indicate what may happen in larger bins and hoppers that have stronger compressive stresses that can cause material to gain strength. The results in Fig. 3 demonstrate that scale (compressive stress) and moisture have substantial impact upon mechanical properties and flow behavior of pine particles. Additional tests were also conducted with different test conditions to investigate the effects of different material fill heights in the hopper and also using boards in the bottom of the hopper to act as gates during the filling process. Those tests are reported in Appendix A Fig. A.4. and Fig.A.5, whichindicate that applying weight to the top of material in the hopper results in a complex stress pattern that does not follow Jenike's assumption.

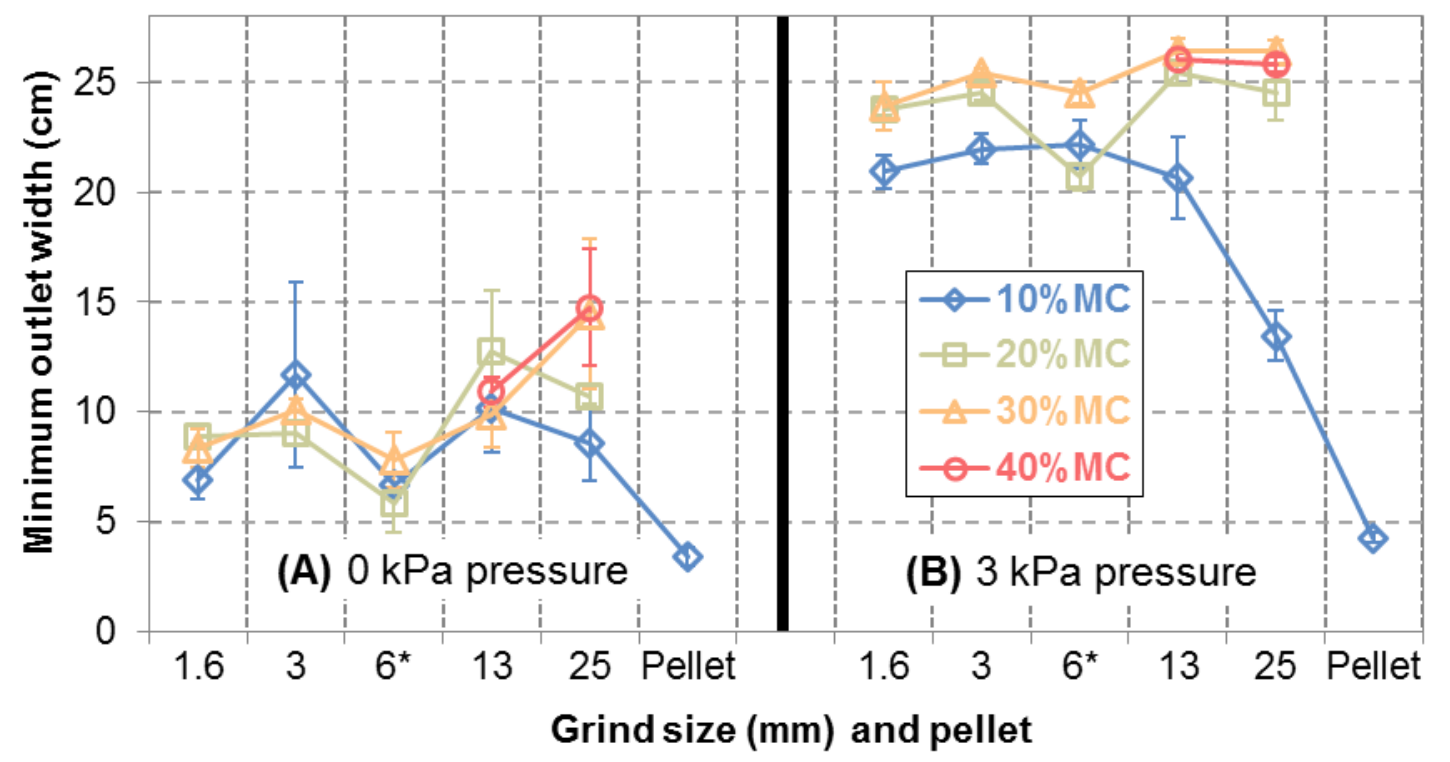

Figure 3. Critical bridging widths of pine samples measured in a hopper with an adjustable outlet. Panel A shows data from experiments without applied pressure, while panel B displays data from the $3 \mathrm{kPa}$ experiments in which force was applied to the top surface of the material.

\subsection{Evaluation of auger Feeder Tests}

As described above, auger feeder tests were conducted separately using different sizes of augers operating at approximately $20 \%$ and $40 \%$ of the maximum rotational velocity (labeled Vol-low and Vol-high, respectively). After the volumetric flow tests were completed, gravimetric tests were also performed that attempted to match the volumetric feed rates (in $\mathrm{kg} / \mathrm{hr}$ ). Panels A and B in Fig. 4 display the average measured feed rates $Q$ for the low speed tests using the small auger using volumetric (Vol-low) and gravimetric modes (Grav-low), respectively. Corresponding data for the other auger feeding tests is shown in Appendix A Figures A.7 through A.9. As intended, the tests conducted in gravimetric mode shown in Panel B closely mimic the tests conducted in volumetric mode, but the repeatability of the tests was much better, resulting in narrower repeatability bars. For the $1.6 \mathrm{~mm}$ grind size, the feed rate decreases with increasing moisture even though the materials with higher moisture have greater densities (see Fig. 4A). For the 13 

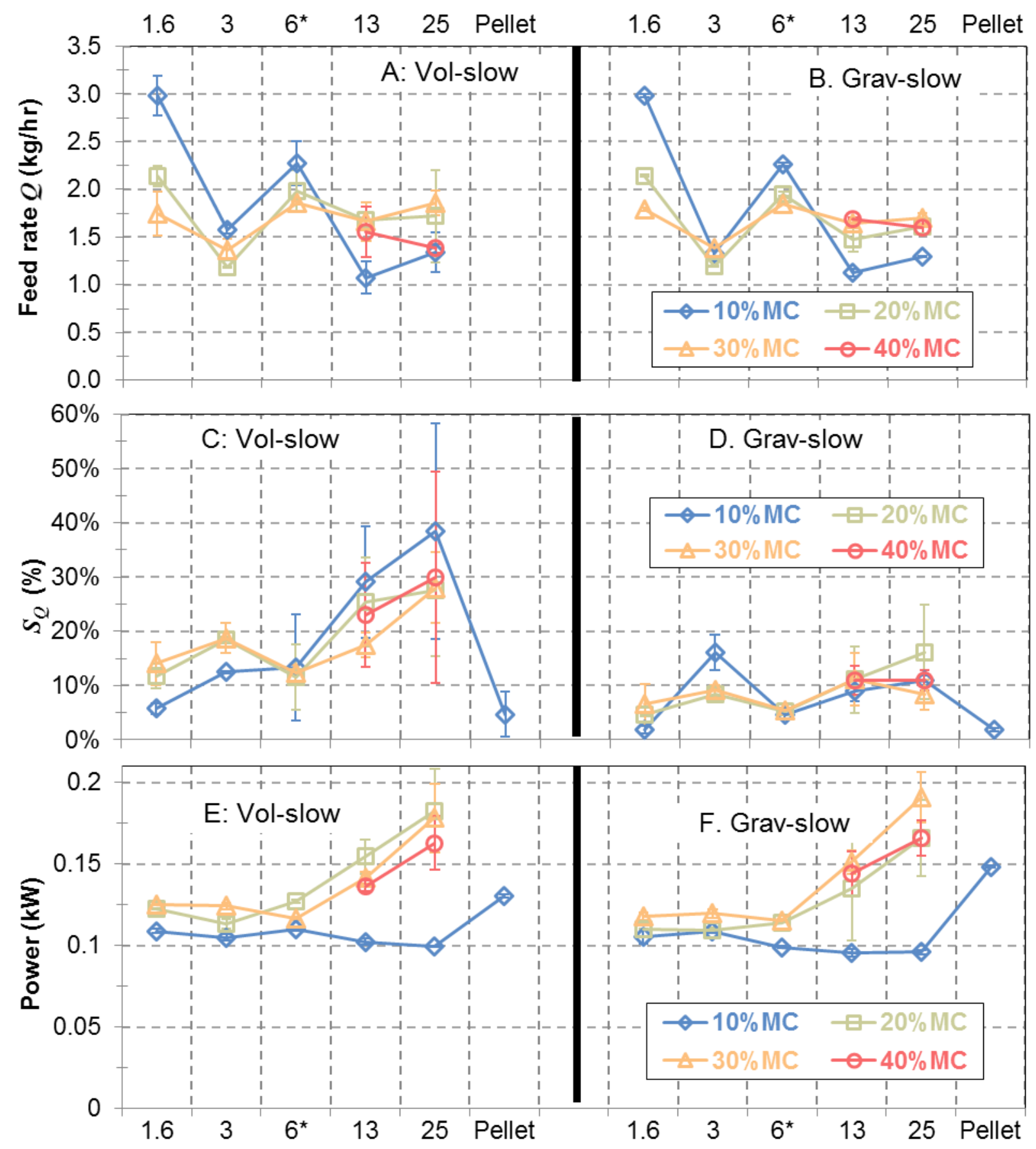

Figure 4. Results from tests with the small auger at low feed rate. Panels A, C and E show the feed rate, the time variability of the feed rate as a percent of the average feed rate and the power consumption, respectively, for the volumetric-mode tests. Panels on the left (B, D and F) show corresponding results from the gravimetric tests. The feed rate of the pellets in Panels A and B (not shown) was 10.8 and $14.6 \mathrm{~kg} / \mathrm{hr}$, respectively. The bars show the difference between repeated tests.

$\mathrm{mm}$ and $25 \mathrm{~mm}$ grind sizes, the effect reverses with the $10 \%$ moisture content (MC) material having the lowest feed rate, as expected based upon density considerations. Interestingly, the 6 
mm knife-ground sample has a much higher feed rate than the $3 \mathrm{~mm}$ and $13 \mathrm{~mm}$ hammer-ground samples, which is likely related to the fact that it has a higher tapped bulk density (Fig. 2A) and a smaller minimum arching width (Fig. 3A).

Panels $\mathrm{C}$ and D display the time variation in the feed rate $S_{Q}$ for the same measurements. For the hammer-ground samples fed in volumetric mode (Panel C, Vol-low), the time variation in the feed rate $S_{Q}$ appeared to increase with particle size and bulk density, except for the $6 \mathrm{~mm}$ knifeground sample which had a lower variation in its feed rate than the $3 \mathrm{~mm}$ grind sample. This observation is consistent with the $6 \mathrm{~mm}$ material being more flowable, allowing it to flow less erratically. The pellet tests, which had feed rates greater than $10 \mathrm{~kg} / \mathrm{hr}$, had the lowest time variability. The time variation in the feed rate for the gravimetric-mode feeding tests (Panel D, Grav-low) was lower than it was for the same tests using volumetric-mode, except the $3 \mathrm{~mm}$ grind at $10 \% \mathrm{MC}$. Panels $\mathrm{E}$ and $\mathrm{F}$ show the power consumption of the motor that drives the feeder during the Vol-low and Grav-low tests, respectively. For materials with $M C \geq 20 \%$, power consumption increases as the grind size increases above $6 \mathrm{~mm}$. The Acrison bin auger feeder is equipped with two stirring augers (see Appendix A Figure A.6), and the increased power consumption for the large, high moisture particles is likely caused by interlocking between the particles in the space between the stirring augers and the bin walls. This effect is so strong that during a test using the $13 \mathrm{~mm}$ grind at $20 \% \mathrm{MC}$ material became wedged between a stirring auger and the bin wall causing the stirring auger (which has a $3.8 \mathrm{~cm}$ diameter steel shaft that is only $46 \mathrm{~cm}$ long) to bend and become inoperable.

Appendix A Fig. A.9 shows similar feed rate and power consumption data for experiments that employed the $63 \mathrm{~mm}$ screw auger operating at the higher feeding rates (approximately $40 \%$ of the maximum feed rate), resulting in feed rates that are approximately 20 times higher than those featured in Fig. 4. In general, the trends for the higher feed rate and larger auger are similar to those displayed in Fig. 4, although using a larger auger decreased the variability in the feed rate to less than $5 \%$ for the $6 \mathrm{~mm}$ grind and smaller materials and to less than approximately $10 \%$ for the $13 \mathrm{~mm}$ and $25 \mathrm{~mm}$ grinds. A limited number of additional tests were also conducted using a $63 \mathrm{~mm}$ diameter ribbon auger (data not shown), and the observed trends were similar to those obtained using the large screw auger. These results indicate that material feed rate and variability in the feed rate in a volumetric auger feeder are dependent upon material (shear) strength as well as bulk density and particle size. Employing a gravimetric feeder can increase control and reduce the variability of the material feed rate, although gravimetric feeders are substantially more complicated to operate and have higher costs.

\subsection{Evaluation of shear tests}

Instantaneous yield loci (IYL) of the materials were measured using preshear compression stresses of $0.5 \mathrm{kPa}, 2.0 \mathrm{kPa}$ and $7 \mathrm{kPa}$. Many of the IYLs from different samples had all of the measured points within a single standard deviation of the points of IYLs for other samples, and such samples were assigned a common designator. Because the standard deviations of the IYLs of a sample can overlap with multiple other samples, some of the samples were assigned multiple designators. All of the key material parameters from the shear tests, including the designators, are shown in Appendix A Table A.1. A total of five unique sample sets, labeled "a" through "e", were identified that had IYLs with non-overlapping standard deviation envelopes at preshear stresses of $0.5 \mathrm{kPa}$ and $2.0 \mathrm{kPa}$. At a preshear of $0.5 \mathrm{kPa}$, only a single sample $(6 \mathrm{~mm} * 30 \mathrm{MC})$ 
was assigned multiple set designators ( $\mathrm{b}$ and c), while at a preshear of $2.0 \mathrm{kPa}$, a total of five samples merited multiple designators. At a preshear of $7.0 \mathrm{kPa}$, all samples fit within three sets, and two samples merited multiple designators. These results show that IYLs of the different samples become more similar as compressive stress increases. For the purposes of data reduction, samples that share the same set of designators are grouped and plotted together. The sample groups for a preshear stress of $2.0 \mathrm{kPa}$ are shown in Fig. 5, and the groups for preshear stresses of $0.5 \mathrm{kPa}$ and $7 \mathrm{kPa}$ are shown in the Appendix A Figures A.10 and A.11. As noted above, the IYL obtained for pellets with a preshear of $0.5 \mathrm{kPa}$ were not reliable. Consequently, additional tests using a preshear stress of $1.0 \mathrm{kPa}$ were performed for pellets and a few other materials (see Appendix A Fig. A.12). Because of the pellet's large particle size and lack of fines, it is not expected that they would perform well in a shear cell. An important feature that all of the IYL share is that when extrapolated to low values of compressive stress, $\sigma$, they all pass near the $(\tau, \sigma)$ origin, which indicates that all of the samples have low cohesion. All of the IYL are also highly linear with only minor downward curvature for some of the samples over the range of pressures tested. To check the performance of the shear cell and the test method, a BCR® certified Reference Material (limestone, Signma Aldrich, 207-439-9) was also tested using preshear stresses of 3 and $6 \mathrm{kPa}$. The shear results are compared with results from obtained from a 27 lab round-robin using limestone powder CRM-116 at the same conditions in Appendix Figures A.13 and A.14. [29]. The results presented here are slightly lower than those from the round-robin but are within or close to 2 standard deviations. Performing a full calibration of the shear tester used for these results did not significantly change the measured results.

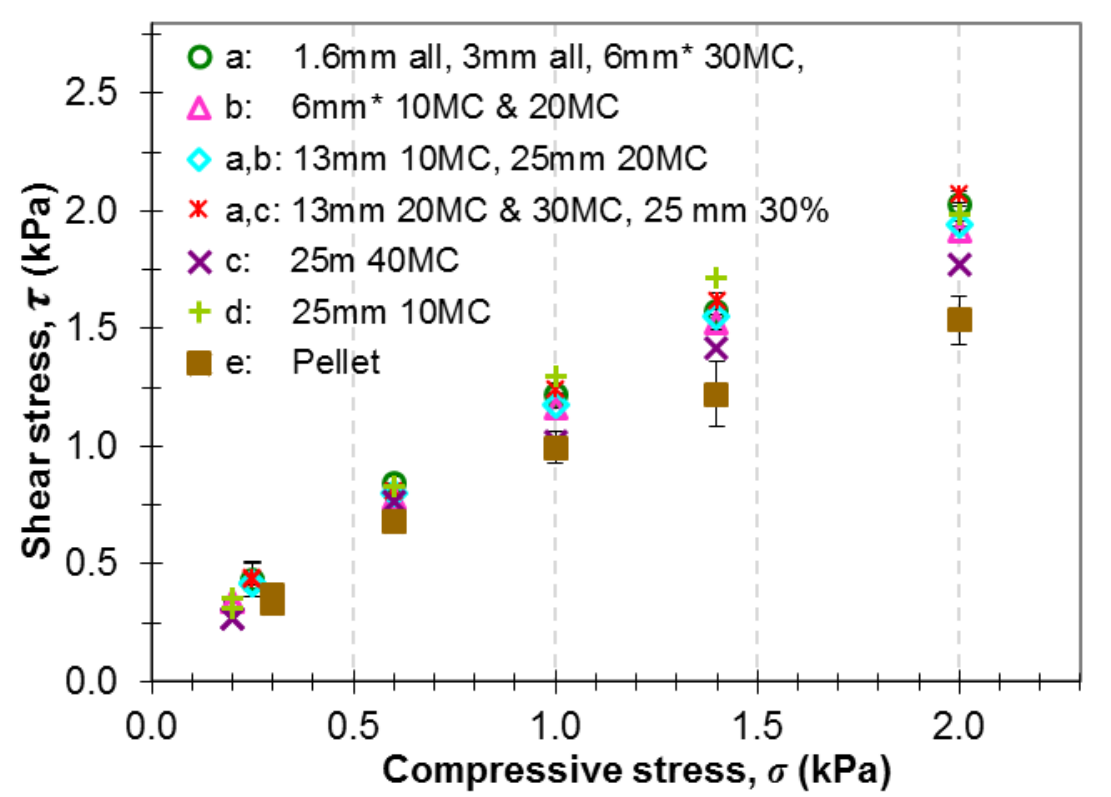

Figure 5. Grouped instantaneous yield loci (IYL) of the woody samples for a preshear compression stress of $2.0 \mathrm{kPa}$. Groups were formed by combining samples according to the set designations in Appendix A Table A.1. Repeatability bars show the standard deviation of all measurements within each group.

Figure 6 shows the material flow functions for the woody materials at $10 \% \mathrm{MC}$ and also includes lines of constant flowability, $f f c$, which is defined as the ratio $\sigma_{1} / f_{c}$. Larger $f f_{c}$ values indicate better flow behavior because material flow occurs when $\sigma_{1}$ overcomes $f_{c}$. Comparing the material 
flow functions with lines of constant flowability, $f f_{c}$, indicates that the ground samples are mostly easy-flowing. The samples with the highest flowability are the pellets, the $6 \mathrm{~mm}$ and $25 \mathrm{~mm}$ grind samples in that order, while the most difficult flowing materials at low compressive stress were the $1.6 \mathrm{~mm}$ and $3 \mathrm{~mm}$ grinds. Note that these results are somewhat in conflict with the hopper and auger feeding tests described above. The material flow functions at higher moisture contents follow similar trends and are shown in Appendix A Figures. A.15 through A.17.

The hopper flow factors $f f$ ranged from 1.24 for the pellets to 1.39 for the $25 \mathrm{~mm}$ grind at $10 \%$ MC. Because the hopper flow factors $f f$ are ratios of compacting stress to shear stress, they can be plotted in the same diagram as the material flow functions. The range of $1 / f f$ measured for all tests without applied pressure is shown as a gray wedge in Fig. 6. For all of the materials, the flow functions appear to intercept the flow factors at very low applied stresses; however, the Schulze ring shear tester is not capable of accurately measuring IYL for preshear stresses lower than approximately $0.5 \mathrm{kPa}$ because of the very low consolidating pressures and shear stresses that are involved as explained above (the lowest consolidating pressures on the IYL curves for a preshear stress of $0.5 \mathrm{kPa}$ is approximately $0.07 \mathrm{kP}$, which is approximately $0.4 \%$ of the full range of the Schulze ring shear tester). In order to determine the intercepts of the material flow functions with the 1/ff curves, it is necessary to extrapolate flow functions from approximately $1.4 \mathrm{kPa}$ to between 0 and $0.7 \mathrm{kPa}$ on the $\sigma_{1}$ axis. As noted above, extrapolating the flow functions greatly increases the uncertainty in predicting the minimum hopper opening [24,26].

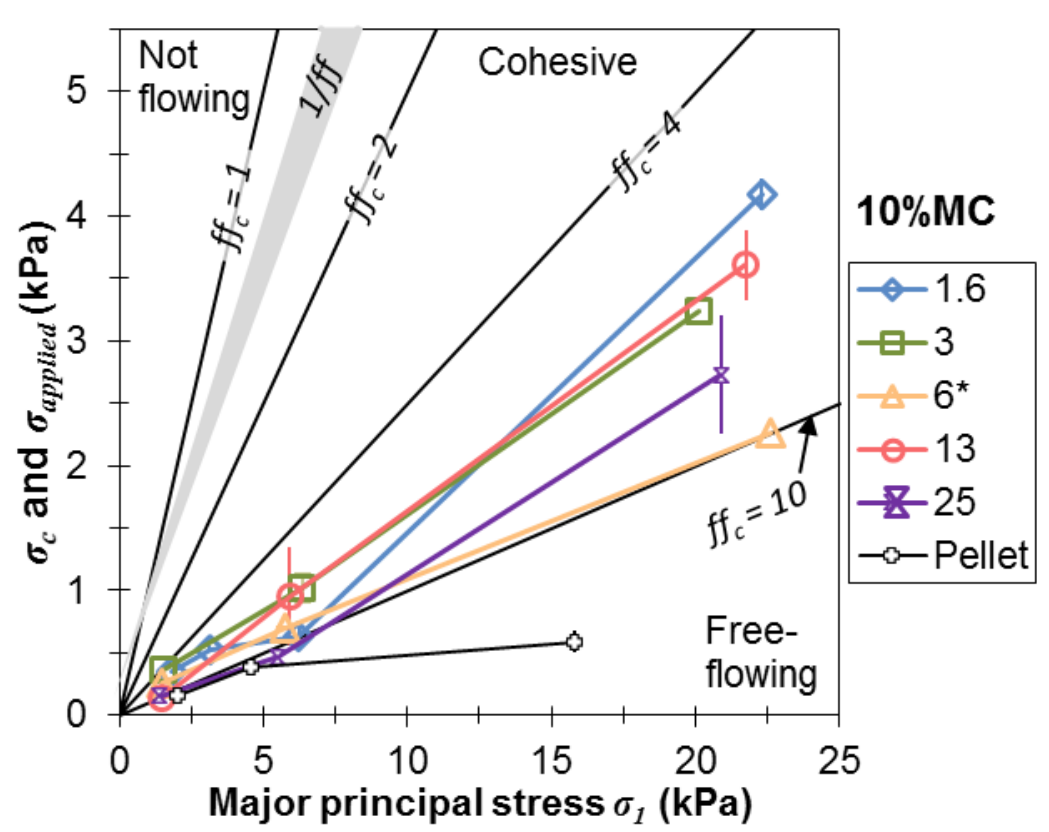

Figure 6. Flow functions of the $10 \% \mathrm{MC}$ samples and pellets. The range of inverse hopper flow factors $(1 / f f)$ is shown as a gray wedge, and lines of constant flowability, $f f_{c}$, are also shown for comparison.

Recently, Barletta and Poletto [17] reported flow function measurements and corresponding hopper tests of two sawdust samples with similar size distributions as the $3 \mathrm{~mm}$ and $6 \mathrm{~mm}$ grinds tested in this work. The moisture contents of their two woody samples were $16 \%$ and $49 \%$. Barletta and Poletto [17] used a manual Schulze ring shear tester for their shear test measurements and reported unconfined yield stress $\left(\sigma_{c}\right)$ measurements of approximately 0.36 
$\mathrm{kPa}$ and $0.54 \mathrm{kPa}$ at a major principal stress $\left(\sigma_{l}\right)$ of approximately $1.5 \mathrm{kPa}$. Those measurements are approximately $35 \%$ and $140 \%$ higher for the wet and dry powders than the values reported here. The same flow measurements appear to have been reported by Miccio et al. [11]. Barletta and Poletto also report well-behaved material flow function curves for $\sigma_{l}$ as low as 0.2 $\mathrm{kPa}$. Presumably, this would have required performing tests with consolidating pressures as low as approximately $0.01 \mathrm{kPa}$, which is an order of magnitude smaller than the instrument's recommended operating range. Schulze and Wittmaier [30] have demonstrated that a Schulze ring shear tester can perform shear tests on highly dispersed dry powders at similar low consolidation stresses; however, special procedures must be followed, and there is still substantial uncertainty. For example, $0.07 \mathrm{kPa}$ corresponds to the normal stress under a layer ground wood powder that is less than $5 \mathrm{~cm}$ in height, so even the location of the shear plane greatly influences the consolidation pressure. For this work, we were unable to obtain reliable results using compressive stresses smaller than $0.07 \mathrm{kPa}$, and so determining the critical arching width required extrapolation of the material flow functions, which Barletta and Poletto did not find necessary.

Oginni et al. [31] reported the physical and flow properties of fractionated loblolly pine grinds with particle sizes ranging from less than $0.25 \mathrm{~mm}$ to $1.4 \mathrm{~mm}$ and moisture contents ranging from $4.8 \%$ to $25 \%$. They used a Brookfield flow tester to measure unconfined yield stress $\left(\sigma_{c}\right)$ at a major principal stress $\left(\sigma_{l}\right)$ as small as $2.2 \mathrm{kPa}$. The material flow functions reported by Oginni et al. are highly linear, and the $0.5 \mathrm{~mm}$ and $0.71 \mathrm{~mm}$ fractions exhibited similar flow properties as the unfractionated material, which had a geometric mean particle diameter of $1.3 \mathrm{~mm}$, making it similar in size to the $6 \mathrm{~mm}$ grind reported in this work. The unconfined yield stresses $\left(\sigma_{c}\right)$ measured by Oginni et al. [31] for the wet and dry powders are approximately $25 \%$ and $40 \%$ higher, respectively, than for similar materials reported here. This difference in reported measurement values is within the range expected for slightly different materials tested using different types of shear testers [20].

Wu et al [32] employed a custom large annular shear tester to measure the shear properties of various dry wood chips and wood pellets (less than 10\% MC) using preshear pressures ranging from 1 to $3.4 \mathrm{kPa}$. The smallest wood chips that they tested had a Sieve 50 of approximately 5 $\mathrm{mm}$, making it similar in size to the $25 \mathrm{~mm}$ grind sample in this work. Wu et al [32] reported an effective angle of internal friction of $53^{\circ}$ for the smallest dry wood chips, which is significantly higher than the value measured for the dry $25 \mathrm{~mm}$ grind in this work, which was $41^{\circ}$ and $38^{\circ}$ for tests conducted using preshear stresses of 0.5 and $2.0 \mathrm{kPa}$, respectively (values are presented in Table A.1 in the Appendix). Comparing the shear results reported in this work with these other published values from similar woody materials indicates that the woody materials in the present report are less cohesive and easier-flowing than the materials in previous publications. Chen et al. reported shear testing of sawdust and two coal samples using a Jenike linear shear tester, which has been shown to be very comparable in terms of results to a Schulze ring shear tester for several materials [33]. The Sieve 50 of the sawdust tested by Chen et al. was approximately 100 microns, so it was finer and had a high bulk density of $300 \mathrm{~kg} / \mathrm{m}^{3}$ at a moisture content of $7 \%$ compared to the samples in this work. The sawdust had a measured flow factor of 1.18 , which is very close to the range reported here, and a negative critical applied stress $\sigma_{\text {crit }}$ of approximately $6 \mathrm{kPa}$ based on extrapolation of the flow functions from $20 \mathrm{kPa}$ of consolidation stress (note that a negative value for $\sigma_{\text {crit }}$ leads to a negative value for $W_{\text {min,pred }}$ based upon Eq. 1, which is 
unrealistic). By comparison, the coal samples exhibited slightly lower effective angles of internal friction and higher flow factors in the range of 1.3 to 1.6. The flow functions of wood grinds reported here are also similar to flow functions of salt, sugar, and corn starch reported by Fitzpatrick et al. [34].

Figure 7A compares the predicted minimum hopper outlet width $\left(W_{\text {min,pred }}\right)$ calculated using the Jenike approach to the measured values $W_{\text {min,meas }}$. The predictions appear reliable and slightly conservative (i.e. the predictions over estimate $W_{\text {min,meas }}$ ) for the $1.6 \mathrm{~mm}$ and $3 \mathrm{~mm}$ grinds at $10 \%$ and $20 \% \mathrm{MCs}$ and also for the $6 \mathrm{~mm}$ grind at $10 \%$ and $20 \% \mathrm{MC}$. It significantly underestimates $W_{\text {min,meas }}$ for all of the $13 \mathrm{~mm}$ grinds and for the $25 \mathrm{~mm}$ grinds at $10 \%$ and $30 \% \mathrm{MC}$. As mentioned above, the error for the $25 \mathrm{~mm}$ grind at $10 \% \mathrm{MC}$ is expected because the $W_{\text {min,meas }}$ is only approximately 9 times that of the geometric mean particle size. For several of the samples, $W_{\text {min,pred }}$ is reported as zero because extrapolating the material flow functions to the hopper flow factor lines resulted in negative estimates for the critical applied stress $\sigma_{c r i t}$, which is not physically realistic. Appendix Figure A.18 plots the same variables as Fig. 7A, except that $\sigma_{\text {crit }}$ was estimated using the hydrostatic assumption. Note that the two figures are nearly identical, indicating that $W_{\text {min,pred }}$ is not sensitive to the assumptions used to estimate $\sigma_{\text {crit. }}$. The results reported here for dry powders are in general agreement with the findings of Barletta and Poletto [17], who reported that $W_{\text {min,pred }}$ for both dry or wet wood powders match $W_{\text {min,meas }}$ quite closely for a hopper with a semi-angle of approximately $30^{\circ}$. The findings here also generally match the results reported by Barletta et. al., who noted that flow predictions for pine wood chips, Scots pine wood powder, and reed canary grass, as predicted using a range of shear testers, substantially overpredicted the size of the hopper outlet needed for reliable flow [35]. On the contrary, though, the results here indicate that for wet powders, $W_{\text {min,pred }}$ may be substantially larger or smaller than $W_{\text {min,meas. }}$ Overall, the correlation between $W_{\text {min,pred }}$ and $W_{\text {min,meas }}$ is quite poor with a coefficient of determination $\left(R^{2}\right)$ of 0.56 . The poor correlation is expected based upon the necessity of extrapolating the material flow functions to very low values of applied stress, which is often necessary for easy-flowing materials [24].

In order to identify patterns in the ability to reliably use shear test data to estimate $W_{\text {min,meas }}$, it is helpful to estimate the uncertainty in the measurement data. The repeatability of measuring the unconfined yield stress $\sigma_{c}$ of the materials appeared to be approximately the same for each of the separate materials, regardless of moisture content or the magnitude of the preshear stress and was approximately 70, 70, 80, 50, 400, and $230 \mathrm{~Pa}$ for the pellets and the $1.6 \mathrm{~mm}, 3 \mathrm{~mm}, 6 \mathrm{~mm}, 13$ $\mathrm{mm}$, and $25 \mathrm{~mm}$ grinds respectively. The upper limit of the $95 \%$ statistical confidence interval for each $\sigma_{c}$ was estimated using

$$
C I_{\sigma c, 95 \%+}=\bar{\sigma}_{c}+t_{95 \%, 5} \frac{s_{\sigma c}}{\sqrt{6}}
$$

where $t 95,5$ is the $t$-statistic corresponding to $95 \%$ confidence with six replicates (equal to 2.57), and $\bar{\sigma}_{c}$ and $s_{\sigma c}$ are the mean and standard deviation, respectively, of the measurements for $\sigma_{c}$ for each sample. The upper limit of the $95 \%$ confidence interval for $\sigma_{c r i t}\left(C I_{\sigma r i t, 95 \%+}\right)$ was determined graphically as described above by replacing the measurements of $\bar{\sigma}_{c}$ with $C I_{\sigma r i t, 95 \%+}$ from Eq. 2 , and this value was then used in Eq. (1) to estimate the upper limit of the 95\% confidence interval

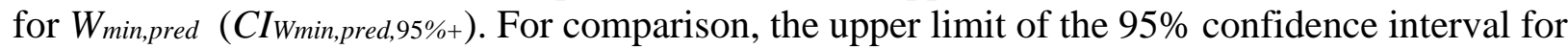
$W_{\text {min,meas }}\left(C I_{\text {Wmin,meas, } 95 \%+}\right)$ was also calculated in the same manner as exemplified by Eq. (2). 
Figure 7B plots $C I_{W m i n, p r e d, 95 \%+}$ as a function of $C I_{W m i n, m e a s, 95 \%+}$. The black line shows the leastsquares regression fit between the variables and has slope of 3.5 and an $R^{2}$ of 0.84 , which indicates that the uncertainties in the hopper measurements are much less than those of the predictions and there is a significant improvement in the correlation between experiment and theory compared to Fig. 7A.
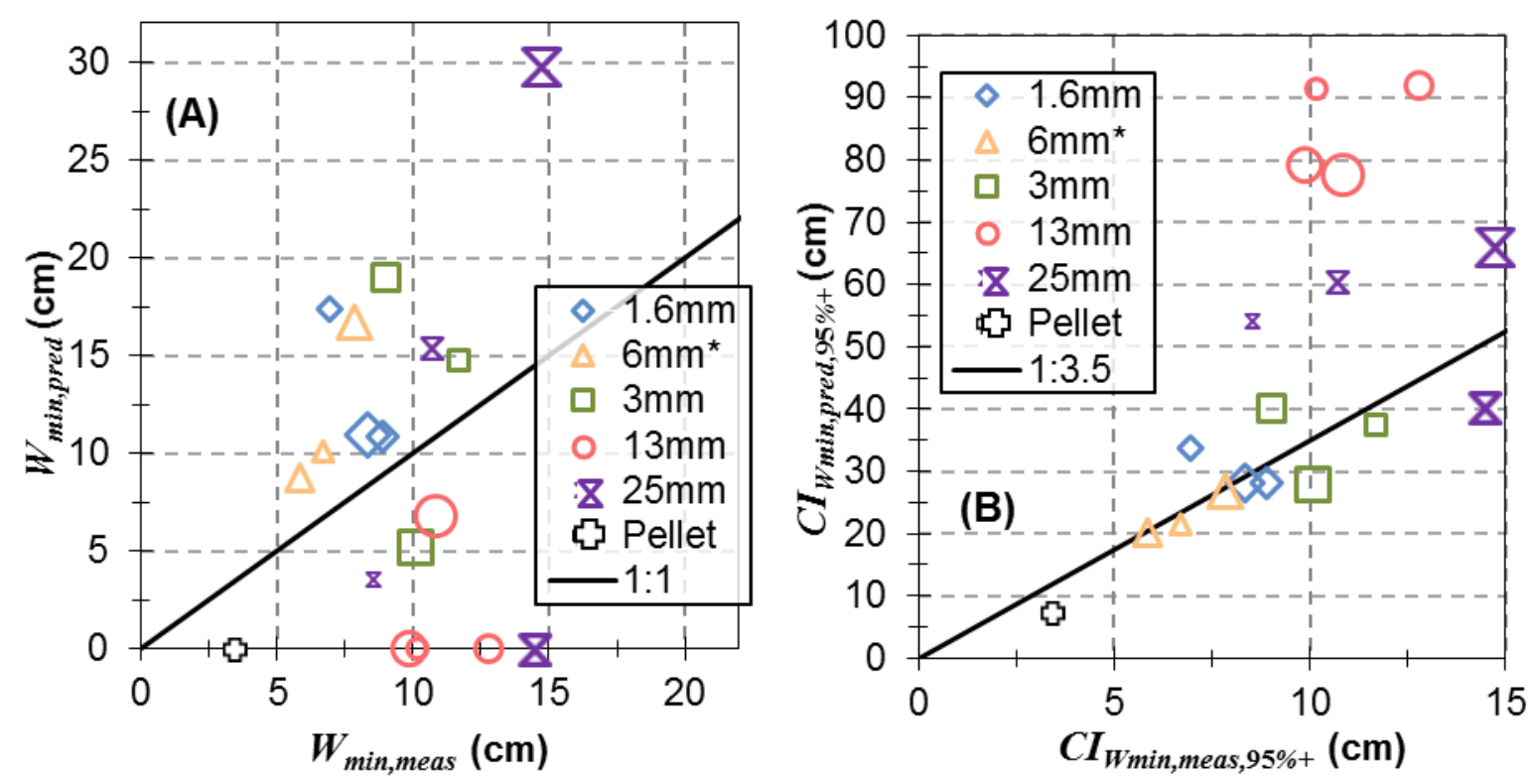

Figure 7. Predicted minimum hopper outlet widths versus the values measured using the hopper tests. Symbol size indicates moisture content with larger symbols representing higher moisture content. Panel A compares the actual prediction to the mean of multiple physical tests, while panel B compares the upper limits of the $95 \%$ confidence intervals of the predictions and physical tests.

The limitations of the shear tester are likely a factor in the large discrepancies between predictions and measured values in Fig. 7. Another possibility for the discrepancy between the $W_{\text {min,pred }}$ and $W_{\text {min,meas }}$ is that the Jenike method applies to hopper flows for which the stress distribution in the material is primarily radial, which is typically the case for powders flowing through a hopper after flow has been initiated and stopped. During the experiments in this work, the hopper was filled and then the outlet was opened without first initiating and stopping the flow, which can result in a primarily vertically oriented stress field. During the hopper tests, it was observed that as the walls were slowly raised, which allowed material at the bottom of the hopper to fall, the top material settled substantially, often more than a centimeter. It is anticipated that the settling of the material caused the compressive stress at the arch of material remaining at the bottom of the walls to shift to a radial stress pattern, in conformance with Jenike's assumptions. While there is no direct evidence for this conjecture, it is clear that shear tests are not suitable for measuring flow properties of these materials at very low compressive pressures because the pressures are outside the accurate operating range of the ring shear tester.

Other hopper flow theories have been developed based upon continuum mechanics to predict the flow of material in hoppers starting with a vertical stress distribution [36,37]; however, due to 
uncertainty in the shear test data at low compressive stresses, pursuing alternate data analysis methods to improve the flow predictions would not likely be helpful. For example, the hopper tests indicate that $W_{\text {min,meas }}$ is approximately 7 and $13 \mathrm{~cm}$, respectively, for the $1.6 \mathrm{~mm}$ and $3 \mathrm{~mm}$ grinds at $10 \% \mathrm{MC}$; however, the IYL for these two materials from the shear tests are identical well within experimental error. These observations lead to the conclusion that much of the discrepancy between $W_{\text {min,meas }}$ and $W_{\text {min,pred }}$ reported here is due to the breakdown of the MohrCoulomb model used in the predictions, which assume that the material consists of rigid and non-fibrous particles [17]. It has been noted that shear tests are often not reliable for materials that exhibit more than approximately $10 \%$ compression or that have a significant content of large particles [20]. The Schulze ring shear tester is considered compatible with materials that have particles as large as $10 \mathrm{~mm}$ if $30 \%$ or more of the particles by mass are smaller than approximately $6 \mathrm{~mm}$. Referring to Table 2, the $13 \mathrm{~mm}$ grind is slightly larger than ideal for the Schulze M shear cell, while the $25 \mathrm{~mm}$ grind is significantly larger than the ideal.

As noted above, it is typically assumed that the critical applied stress in hoppers $\left(\sigma_{c r i t}\right)$ ranges from $1.5 \mathrm{kPa}$ for $100 \mathrm{~L}$ containers (such as the custom hopper used in this work) to $7 \mathrm{kPa}$ for medium and large silos [24]. Guan and Zhang applied compaction pressures ranging from approximately $2 \mathrm{kPa}$ to $30 \mathrm{kPa}$ to wheat flour in a bin/hopper system to determine the impact of applied stress on material strength and flow [38]. After applying the pressure using an instrumented load frame, they dissembled the bin from the bottom up by removing detachable sections to determine $W_{\text {min,meas }}$. They found that $W_{\text {min,meas }}$ increased as the compaction pressure increased from $2 \mathrm{kPa}$ to $5 \mathrm{kPa}$ but did not increase further with further increases in compaction pressure. Westover et al. [18] used the same custom hopper featured in this work to show that $W_{\text {min,meas }}$ increases with compaction pressure for chopped switchgrass but not for ground switchgrass. Figure 3 above also showed that increasing the compaction pressure to $3 \mathrm{kPa}$ increased $W_{\text {min,meas }}$ for all samples, except the pellets and the $25 \mathrm{~mm}$ grind at $20 \% \mathrm{MC}$. However, the shear test data do not exhibit this phenomenon. The predictions shown in Fig. 7 are based upon inverse hopper flow factors, such as those shown in Fig. 6, that were measured using a preshear stress of $0.5 \mathrm{kPa}$. The calculations were also repeated using material properties measured with a preshear stress of $2.0 \mathrm{kPa}$, and practically identical results were obtained (shown in Appendix A Fig. A.19), which is expected because measured values of the effective angle of internal friction have only a small dependence upon the preshear stress (see Appendix A Figures A.20-22). As stated above, the compressible and anisotropic nature of pine particles is likely a cause of the discrepancy between $W_{\text {min,meas }}$ and $W_{\text {min,pred }}$ as reported here because these particles do not meet the assumptions of the Mohr-Coulomb model used in the predictions [17].

\section{Conclusions}

This work has reported rheological characterization of hammer- and knife-milled lodge pole pine as a function of grind size and moisture content. Characterizations included particle size and shape distributions, bulk density, compressibility at $10 \mathrm{kPa}$ uniaxial pressure, elastic recovery from $10 \mathrm{kPa}$ uniaxial pressure, shear strength, effective angle of internal friction, arching in a custom hopper, and feeding performance in volumetric and gravimetric auger feeders. Particle analysis indicates that the width and length distributions of the $3 \mathrm{~mm}$ hammer-ground and the 6 mm knife-ground materials are quite similar, and all the materials have relatively short aspect ratios (<4). Compressibility and elastic recovery increased as much as $15 \%$ and $4 \%$, respectively with increasing MC. Adding $3 \mathrm{kPa}$ of pressure increased the critical arching width for all hopper 
arching tests, except for pellets. Interestingly, for the materials with smaller particles, the average volumetric feed $Q$ rate tends to decrease as moisture increases even though the density of the material increases with moisture content and the auger speeds are the same. Presumably, the decrease in $Q$ is associated with higher particle cohesion, which decreases the material fill factor in the auger housing. This conclusion is supported by the hopper flow tests that indicate that the critical arching width increases with moisture content for both uncompacted and compacted tests. In the auger feeding tests, the time variability of volumetric feed rate $S_{Q}$ increased dramatically with particle size, but the impact of moisture content upon $S_{Q}$ was not as clear. For nearly all tests, gravimetric auger feeding resulted in better feeding control and substantially decreased time variability in the material feed rate.

The shear tests indicate that material strength generally decreased with increasing particle size, although the impact of MC was not entirely consistent. Predictions of the minimum hopper outlet width required to ensure flow $W_{\text {min,pred }}$ appear to suffer dramatically from insufficient force resolution of the shear tests at low applied consolidation stresses, resulting in poor agreement between predictions and measurements. Shear test data further indicated that the materials have low cohesion and that $W_{\text {min,pred }}$ does not significantly increase with increasing applied consolidation pressure, which is contrary to the experimental results using a custom hopper. This discrepancy between the predictions and experiments is likely due to the breakdown of the Mohr-Coulomb flow model, which is not necessarily accurate for compressible and anisotropic particles.

\section{Acknowledgements}

This manuscript has been authored by Battelle Energy Alliance, LLC under Contract No. EE0001034 with the U.S. Department of Energy. The United States Government retains and the publisher, by accepting the article for publication, acknowledges that the United States Government retains a nonexclusive, paid-up, irrevocable, world-wide license to publish or reproduce the published form of this manuscript, or allow others to do so, for United States Government purposes.

\section{Appendix A. Supplementary data}

\section{Supplementary data to this article can be found online.}

\section{References}

[1] D. Carpenter, T.L. Westover, S. Czernik, W. Jablonski, Biomass feedstocks for renewable fuel production: a review of the impacts of feedstock and pretreatment on the yield and product distribution of fast pyrolysis bio-oils and vapors. Green Chemistry, 16(2) (2014), 84- 406.

[2] P.S. Lam, S. Sokhansanj, Engineering Properties of Biomass. Engineering and Science of Biomass Feedstock Production and Provision (pp. 17-35). Springer New York, 2014.

[3] C.L. Williams, T.L. Westover, R.M. Emerson, J.S. Tumuluru, C. Li, Sources of Biomass Feedstock Variability and the Potential Impact on Biofuels Production. BioEnergy Research, 9 (2016) 1-14.

[4] E.W. Merrow, Problems and progress in particle processing, Chem. Innov. Jan. (2000) 3541. 
[5] T.L.Westover, FY14-Q1 1.2.1.3.ML.1 INL Biomass Feeding Survey Report, INL External Report 14-31687, April 2014.

[6] P.D. Jensen, J.E. Mattsson, P.D. Kofman, A. Klausner, Tendency of wood fuels from whole trees, logging residues and roundwood to bridge over openings. Biomass and bioenergy, 26(2) (2004), 107-113.

[7] S. Hinterreiter, H. Hartmann, P. Turowski, Method for determining bridging properties of biomass fuels - experimental and model approach. Biomass conversion and Biorefinery, 2(2) (2012), 109-121.

[8] F. Miccio, N. Silvestri, D. Barletta, M. Poletto, Characterization of woody biomass flowability. Chem. Engin. Trans., 24 (2011), 643-648.

[9] J.E. Mattsson, P.D. Kofman, Influence of particle size and moisture content on tendency to bridge in biofuels made from willow shoots. Biomass and bioenergy, 24(6) (2003), 429435.

[10] J.E. Mattsson, Basic handling characteristics of wood fuels: angle of repose, friction against surfaces and tendency to bridge for different assortments. Scandinavian Journal of Forest Research, 5(1-4) (1990), 583-597.

[11] F. Miccio, D. Barletta, M. Poletto, Flow properties and arching behavior of biomass particulate solids. Powder technology, 235 (2013), 312-321.

[12] K.L. Kenney, W.A. Smith, G.L. Gresham, T.L. Westover, Understanding biomass feedstock variability. Biofuels, 4(1) (2013), 111-127

[13] K. Tannous, P.S. Lam, S. Sokhansanj, J.R. Grace, Physical properties for flow characterization of ground biomass from Douglas Fir Wood. Particulate Science and Technology, 31(3) (2013), 291-300

[14] Q. Guo, X. Chen, H. Liu, Experimental research on shape and size distribution of biomass particle. Fuel, 94 (2012), 551-555.

[15] E. Searcy, J.R. Hess, J. Tumuluru, L. Ovard, D.J. Muth, E. Trømborg, M. Wild, M. Deutmeyer, L. Nikolaisen, T. Ranta, R. Hoefnagels, Optimization of biomass transport and logistics. International Bioenergy Trade 2014 (pp. 103-123). Springer Netherlands.

[16] M. Gil, D. Schott, I. Arauzo, E. Teruel, Handling behavior of two milled biomass: SRF poplar and corn stover. Fuel Processing Technology, 112 (2013), 76-85.

[17] D. Barletta, M. Poletto, An assessment on silo design procedures for granular solid biomass, Chem. Engin. Trans. 32 (2013), 2209-2214.

[18] T.L. Westover, M. Phanphanich, J.C. Ryan, Comprehensive rheological characterization of chopped and ground switchgrass. Biofuels, 6(5-6) (2015), 249-260.

[19] A.W. Jenike, Storage and flow of solids. Salt Lake City, Utah: University of Utah Bulletin Number 123, 1964.

[20] J. Schwedes, Review on testers for measuring flow properties of bulk solids. Granular matter, 5(1) (2003), 1-43.

[21] F. Miccio, A. Landi, D. Barletta, M. Poletto, Preliminary assessment of a simple method for evaluating the flow properties of solid recovered fuels. Particulate Science and Technology, 27(2) (2009), 139-151.

[22] T.L. Westover, A. Matthews, Particle size and shape distributions of hammer milled pine INL/External Report 15-35033, December 2014.

[23] ASTM E873-82(2006), Standard Test Method for Bulk Density of Densified Particulate Biomass Fuels, ASTM International, West Conshohocken, PA, 2006, www.astm.org. 
[24] D. Schulze, Flow properties testing with ring shear testers RST-01.01, RST-01.pc, and RST-XS, 1.6, Schüttgutmesstechnik, Wolfenbüttel, Germany; 2001 (this is a supplemental operator manual for the RST-01.pc).

[25] D. Schulze, RST-CONTROL 95, Schüttgutmesstechnik, Wolfenbüttel, Germany; 2001 (supplemental operator manual for the RST-01.pc).

[26] D. Schulze, Ring shear tester RST-01.pc, Schüttgutmesstechnik, Wolfenbüttel, Germany; 2001 (operator manual for the RST-01.pc).

[27] D. Schulze, Powder and bulk solids. Berlin, Germany, Springer Press; 2008.

[28] G.G Chase, 2001. Solids Notes 10. University of Akron. Available at: https://www.inti.gob.ar/cirsoc/pdf/silos/SolidsNotes10HopperDesign.pdf (accessed Jan. 16, 2017).

[29] D. Schulze, Round robin test on ring shear testers, Adv. Powd. Technol. (2011) 197-202.

[30] D. Schulze, A. Wittmaier, Flow properties of highly dispersed powders at low consolidation stresses, Chem. Eng. Technol. 26 (2003) 133-137.

[31] O. Oginni, O. Fasina, S. Adhikari, J. Fulton, Physical and flow properties of fractionated loblolly pine grinds, Trans. ASABE 59(5) (2016) 999-1008.

[32] M.R. Wu, D.L Schott, G. Lodewijks, Physical properties of solid biomass. Biomass and Bioenergy 35 (2011) 2093-2105.

[33] P. Chen, Z. Yuan, X. SHen, Y. Zhang, Flow properties of three fuel powders. Particuology (2012) 438-443.

[34] J.J. Fitzpatric, S.A. Barringer, T. Iqbal, Flow property measurement of food powders and sensitivity of Jenike's hopper design methodology to the measured values. J. Food Engin. 61 (2004) 399-405.

[35] D. Barletta, R.J. Berry, S.H. Larsson, T.A. Lestander, M. Poletto, Á. Ramírez-Gómez, Assessment on bulk solids best practice techniques for flow characterization and storage/handling equipment design for biomass materials of different classes. Fuel Proc. Technol. 138 (2015) 540-554.

[36] A. Drescher, A.J. Waters, C.A. Rhoades, Arching in hoppers: I. Arching theories and bulk material flow properties. Powder Technol. 84 (1995) 165-176.

[37] G.G. Enstad, The ultimate critical outlet width for flow in mass flow hoppers: theoretical and experimental studies, Internat. J. of Bulk Solids Storage In Silos 1(4) (1985), 9-18.

[38] W. Guan, Q. Zhang, The effect of moisture content and compaction on the strength and arch formation of wheat flour in a model bin. J. Food Engineer. 94 (2009, 227-232. 


\section{Appendix A. Supplementary data}

Table A.1. Key material properties and set designators determined using shear tests.

\begin{tabular}{|c|c|c|c|c|c|c|c|c|c|}
\hline & & & & & & & \multicolumn{3}{|c|}{ IYL Sets } \\
\hline & $\theta_{\min }$ & $\delta_{e f f}$ & $\theta_{\text {exp }}$ & $f f$ & $\tau_{\text {crit }}$ & $W_{\min }$ & $0.5 \mathrm{kPa}$ & $2.0 \mathrm{kPa}$ & $7.0 \mathrm{kPa}$ \\
\hline $1.6 \mathrm{~mm} \mathrm{10MC}$ & 19 & 45.2 & 32.1 & 1.31 & 0.27 & 17.39 & $\mathrm{a}$ & $a$ & $\mathrm{a}$ \\
\hline $1.6 \mathrm{~mm} \mathrm{20MC}$ & 17 & 45.6 & 32.1 & 1.30 & 0.17 & 10.93 & $b$ & $\mathrm{a}$ & $\mathrm{a}$ \\
\hline $1.6 \mathrm{~mm} 30 \mathrm{MC}$ & 19 & 46.2 & 32.1 & 1.28 & 0.18 & 10.86 & $a$ & $a$ & $a, b$ \\
\hline $3 \mathrm{~mm} 10 \mathrm{MC}$ & 14 & 45.6 & 32.1 & 1.30 & 0.18 & 14.87 & $b$ & $\mathrm{a}$ & $b$ \\
\hline $3 \mathrm{~mm} 20 \mathrm{MC}$ & 19 & 46.0 & 32.1 & 1.29 & 0.24 & 19.06 & $b$ & $a$ & $a, b$ \\
\hline $3 \mathrm{~mm} 30 \mathrm{MC}$ & 27 & 46.0 & 32.1 & 1.29 & 0.07 & 5.10 & a & $a$ & a \\
\hline $6 \mathrm{~mm}^{*} 10 \mathrm{MC}$ & 16 & 44.0 & 32.1 & 1.34 & 0.14 & 10.11 & c & $b$ & $b$ \\
\hline $6 \mathrm{~mm}^{*} 20 \mathrm{MC}$ & 33 & 44.3 & 32.1 & 1.33 & 0.13 & 8.81 & c & $b$ & $b$ \\
\hline $6 \mathrm{~mm}^{*} 30 \mathrm{MC}$ & 25 & 45.6 & 32.1 & 1.30 & 0.25 & 16.75 & $b, c$ & $a$ & $b$ \\
\hline $13 \mathrm{~mm} \mathrm{10MC}$ & 12 & 44.7 & 32.1 & 1.32 & 0.00 & 6.18 & $\mathrm{c}$ & $a, b$ & $a$ \\
\hline $13 \mathrm{~mm} 20 \mathrm{MC}$ & 14 & 46.5 & 32.1 & 1.28 & 0.01 & 0.80 & $d$ & a.c & $a$ \\
\hline $13 \mathrm{~mm} 30 \mathrm{MC}$ & 27 & 45.1 & 32.1 & 1.31 & 0.03 & 2.42 & c & a.c & $a$ \\
\hline $13 \mathrm{~mm} 40 \mathrm{MC}$ & 22 & 45.6 & 32.1 & 1.30 & 0.11 & 6.63 & a & a & a \\
\hline $25 \mathrm{~mm} \mathrm{10MC}$ & 14 & 41.7 & 32.1 & 1.39 & 0.05 & 3.55 & $d$ & $d$ & $\mathrm{a}$ \\
\hline $25 \mathrm{~mm} 20 \mathrm{MC}$ & 17 & 44.8 & 32.1 & 1.32 & 0.20 & 14.97 & $d$ & $a, b$ & $a$ \\
\hline $25 \mathrm{~mm} 30 \mathrm{MC}$ & 29 & 45.5 & 32.1 & 1.30 & 0.01 & 0.66 & c & $a, c$ & $\mathrm{a}$ \\
\hline $25 \mathrm{~mm} 40 \mathrm{MC}$ & 30 & 45.9 & 32.1 & 1.29 & 0.53 & 29.71 & c & c & a \\
\hline Pellets & 30 & 38.1 & 32.1 & 1.24 & 0.31 & 6.20 & e & $\mathrm{e}$ & c \\
\hline
\end{tabular}

$\theta_{\min }=$ minimum theoretical hopper semi-angle from vertical; $\delta=$ effective angle of internal friction; $\theta_{\exp }=$ hopper semi-angle used in experiments; $f f=$ flow factor; $\tau_{\text {crit }}=$ predicted critical applied stress in hopper at maximum arch; $W_{\min }=$ predicted minimum hopper outlet width to ensure consistent flow.

Table A.2. Angle of kinematic wall friction.

\begin{tabular}{|c|c|c|c|c|}
\hline Sample & $10 \% M C$ & $20 \% M C$ & $30 \% M C$ & $40 \% M C$ \\
\hline Pellet & 30.0 & \#N/A & \#N/A & \#N/A \\
$25 \mathrm{~mm}$ & 20.7 & 24.0 & 30.3 & 29.2 \\
$13 \mathrm{~mm}$ & 12.8 & 13.5 & 27.4 & 24.0 \\
$6 \mathrm{~mm} *$ & 29.0 & 23.6 & 20.2 \\
$3 \mathrm{~mm}$ & 14.8 & 18.9 & 23.1 & 20.7 \\
$1.6 \mathrm{~mm}$ & 13.8 & 19.9 & 16.2 \\
\hline
\end{tabular}




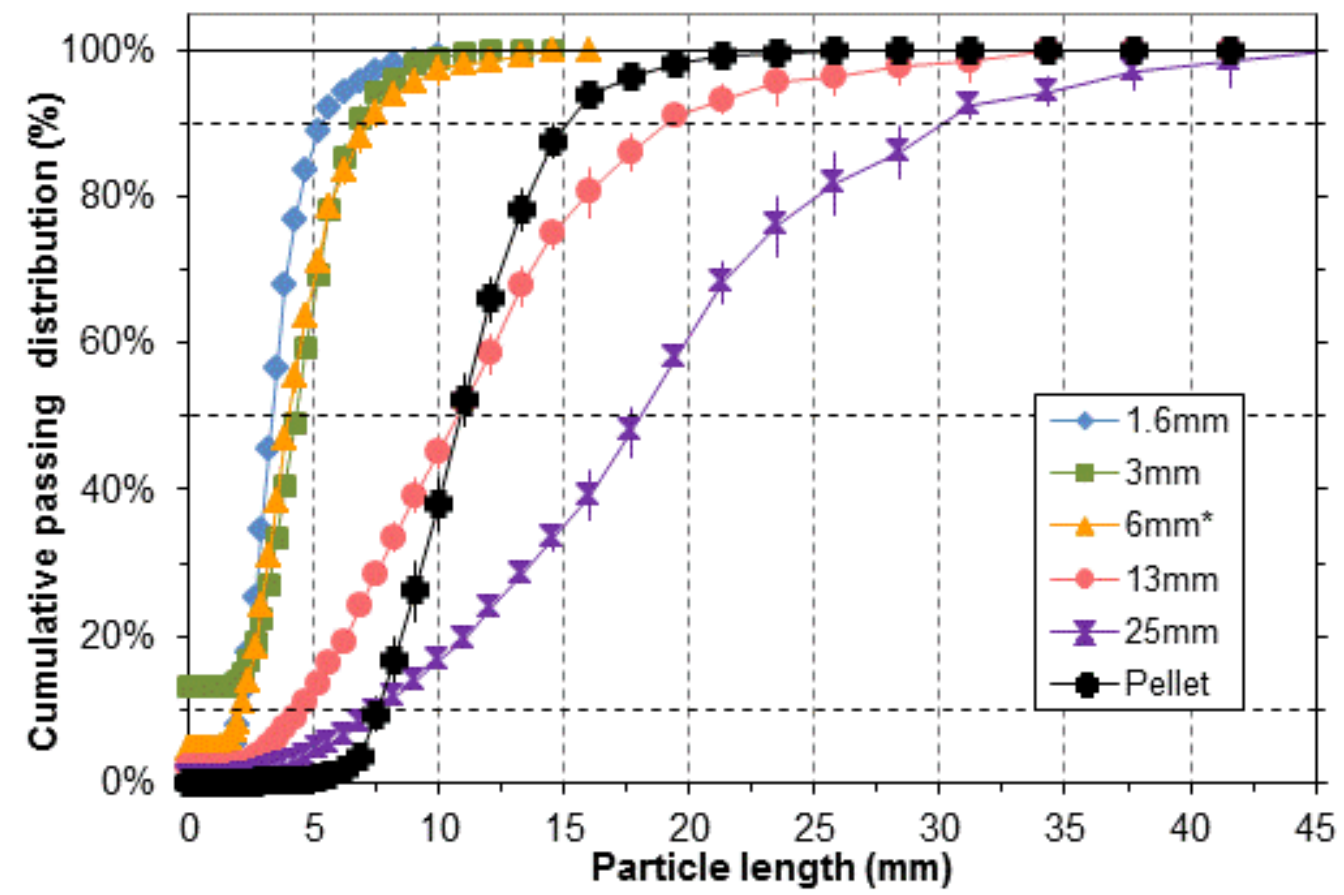

Figure A.1. Cumulative particle length distributions (CPDs) for ground pine samples determined by camera imaging. Repeatability bars, which are often smaller than the symbols, indicate the standard deviation of multiple replicates. 


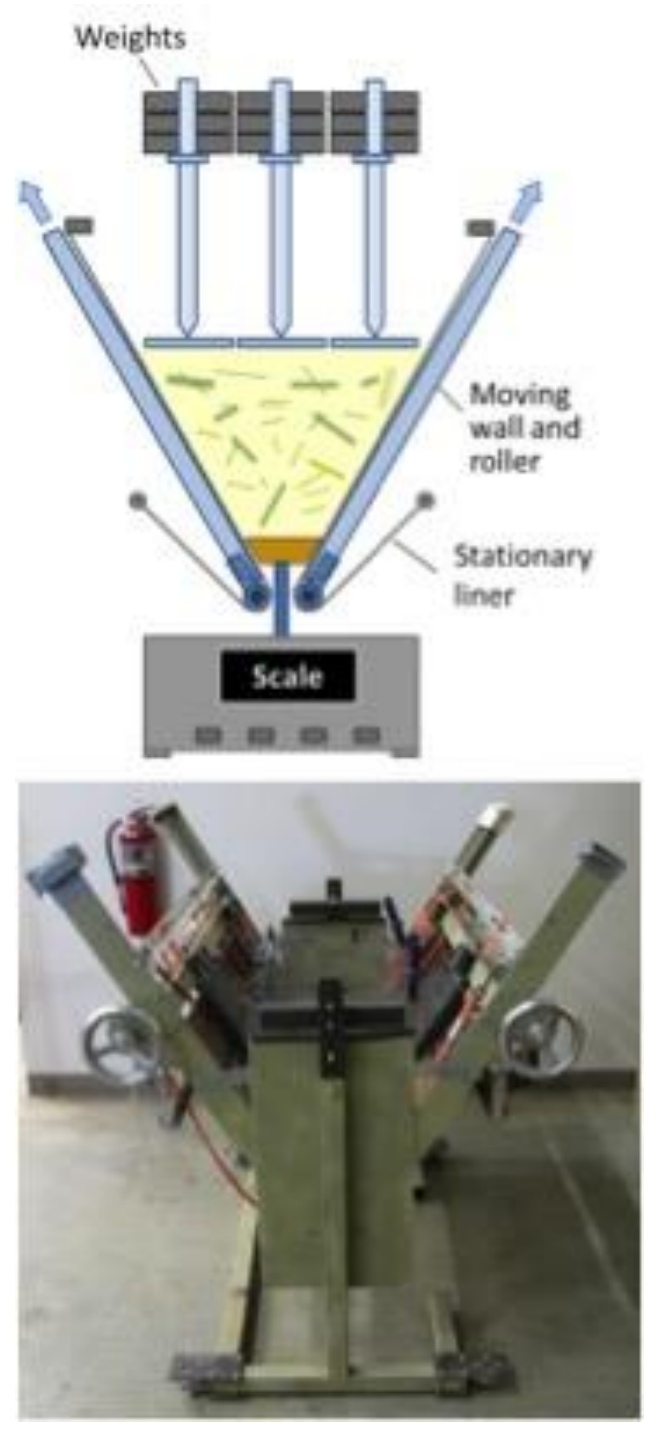

Figure A.2. Schematic and photograph of the custom flow hopper.

Additional hopper notes: After loading material into the hopper, the initial stress state is approximately vertical. As the walls slide upward, material at the bottom of the hopper falls free successively forming new arches at the lower edge of the sliding walls. The release of material relaxes the vertical stress state, such that the material above the arch settles, establishing a radial stress profile, similar to that postulated by Andrew Jenike in his flow function approach. Jenike's approach has been proven to be applicable to flow behavior in a wide range of hopper applications; consequently, the hopper experiments reported without weights are also expected to be applicable in situations in which the weight of material above the arch does not substantially affect the flow. In such situations, the hopper tests using weights to compress the material indicate the potential gain in strength due to the weight of material from above. We admit that these results are not definitive but instead are intended to better understand the research requirements and gauge the merits of a particular solution approach.

Tests have been carried out for a variety of samples and test conditions, including the scenario described above. If a board is placed in the bottom of the hopper that is slightly wider than 
$W_{\text {min,meas, }}$ and then material filled above the board, material does flow after the walls are raised and the board falls free. The methodology for such tests was previously published open-access in Westover, Phanphanich, Ryan, Biofuels 2015; 6(5-6), 249-260. In those tests, the initial flow is particularly rapid because the initial stress state of material in the hopper is approximately vertical, such that the material can flow without arch formation.

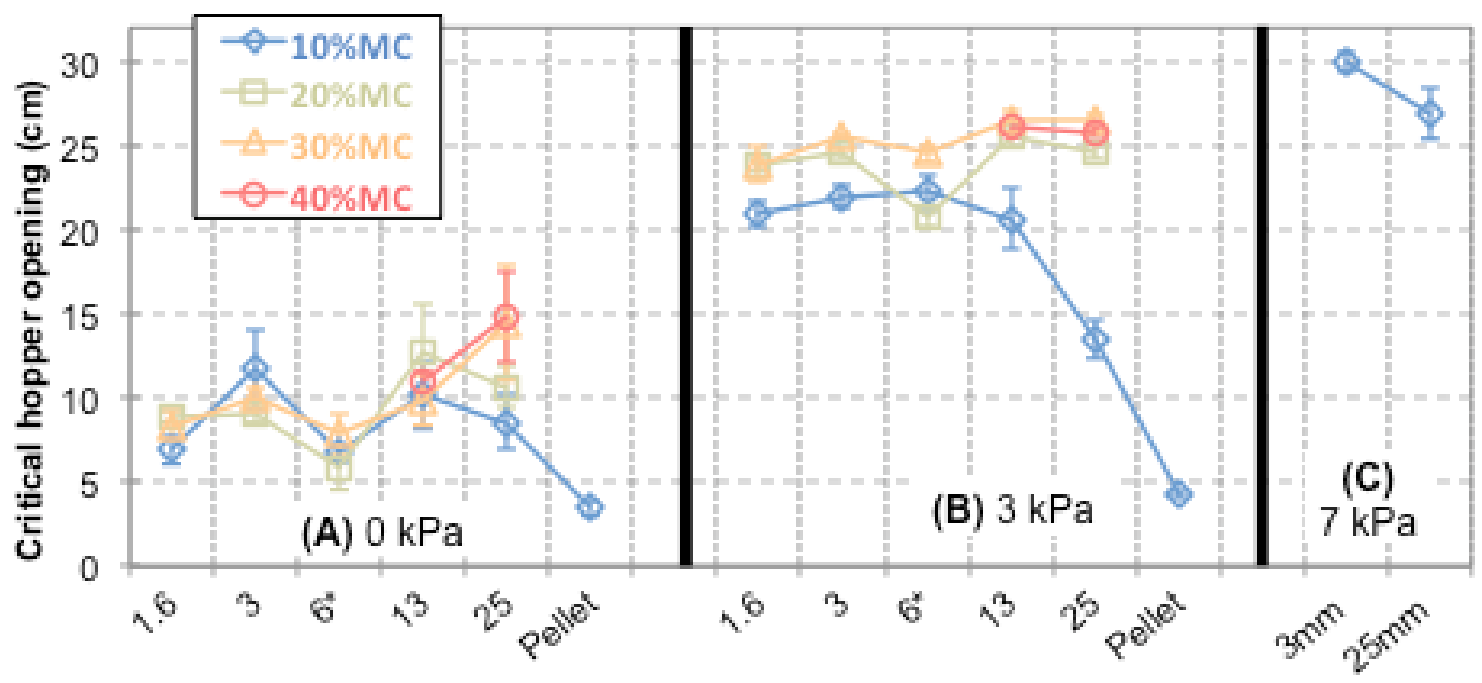

Figure A.3. Same as Fig. 3, except that it includes additional tests conducted with $7 \mathrm{kPa}$ of applied pressure. Applying more pressure continues to increase $W_{\text {min,meas }}$ for the $3 \mathrm{~mm}$ and 25 $\mathrm{mm}$ grinds with $10 \% \mathrm{MC}$.

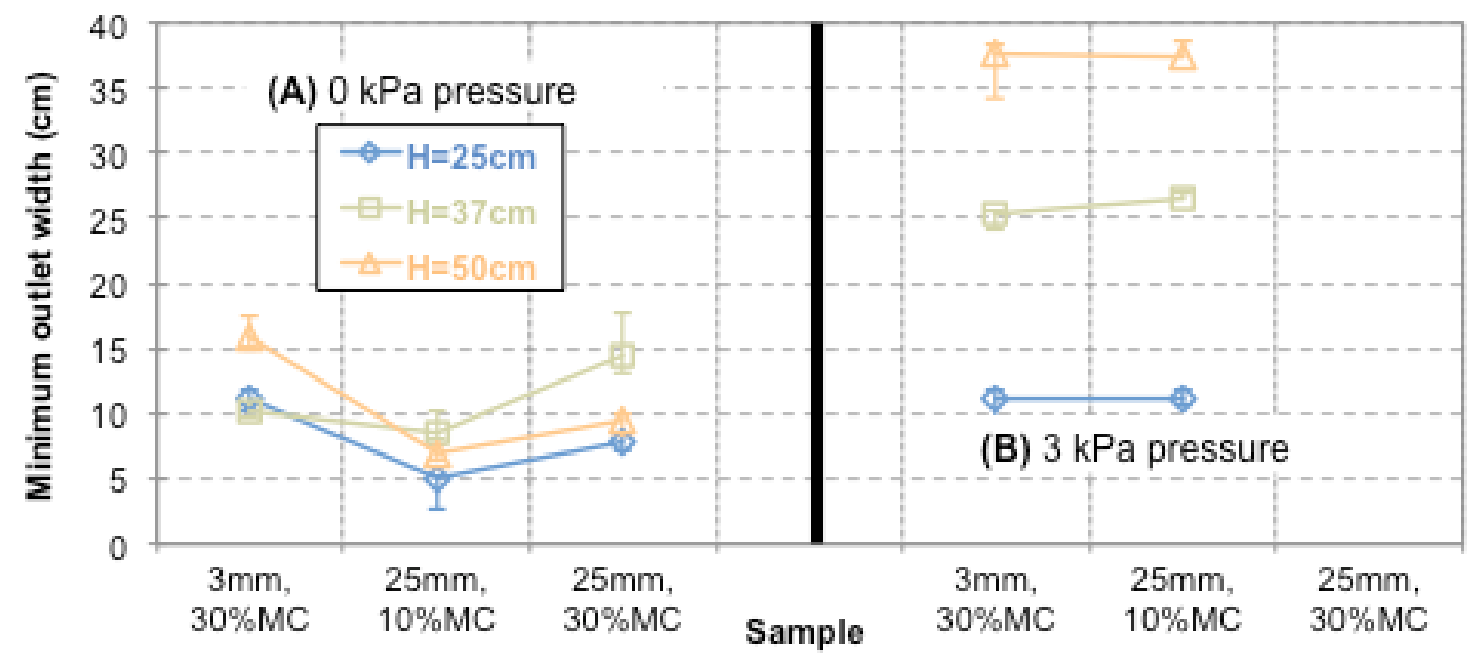

Figure A.4. Critical bridging widths of pine grinds measured in a hopper with an adjustable outlet. Panel A shows data from experiments without applied pressure, while panel B displays data from the $3 \mathrm{kPa}$ experiments in which force was applied to the top surface of the material. For these tests the hopper was filled with different depths of material equal to 25,37 , and $50 \mathrm{~cm}$, respectively. Note that fill level does not have a consistent strong effect for the unweighted tests. For tests in which $3 \mathrm{kPa}$ was applied to the top of the material, $W_{\text {min,meas }}$ increases rapidly with increasing fill level. This behavior is expected because as the amount of material in the hopper 
increases, more material is available to transfer the load to the hopper walls over a greater area, which reduces the applied shear stress. These tests indicate that applying weight to the top of material in the hopper results in a complex stress pattern that does not follow Jenike's assumption.

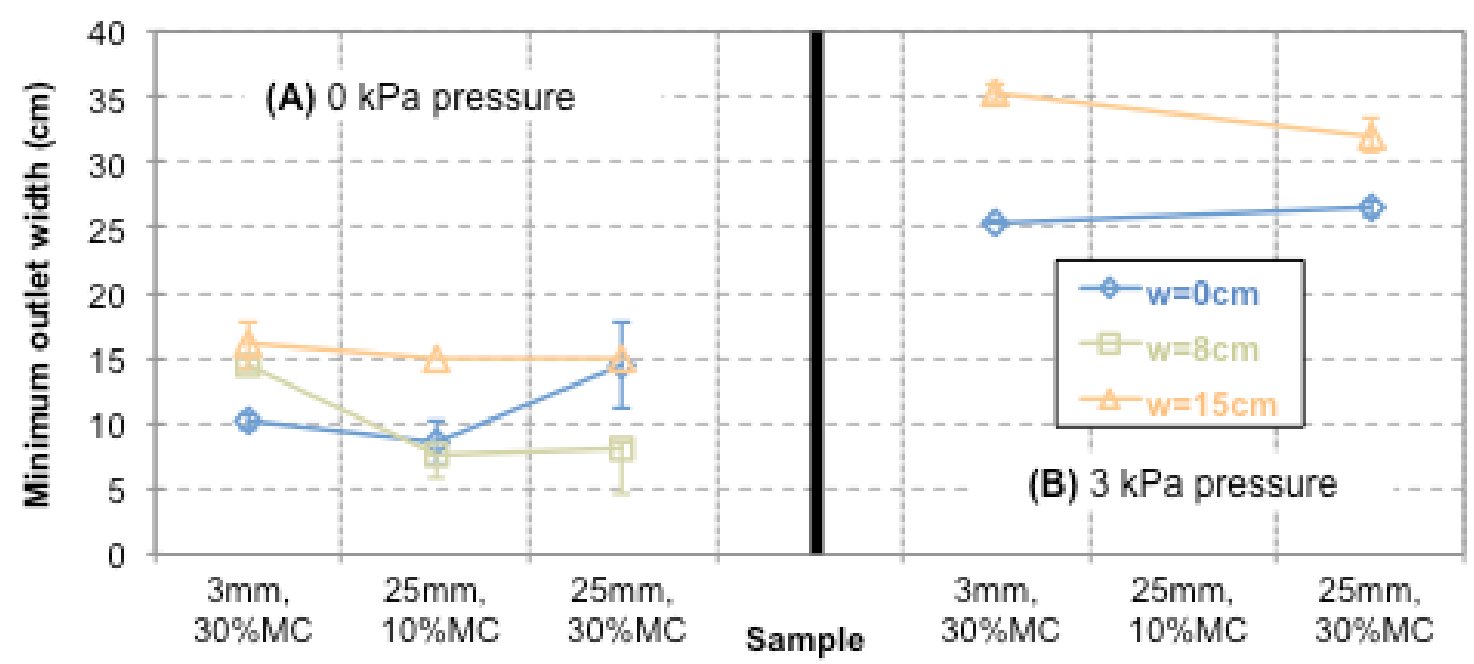

Figure A.5. Critical bridging widths of pine grinds measured in a hopper with an adjustable outlet. Panel A shows data from experiments without applied pressure, while panel B displays data from the $3 \mathrm{kPa}$ experiments in which force was applied to the top surface of the material. For these tests, boards of either $8 \mathrm{~cm}$ or $15 \mathrm{~cm}$ were placed in the bottom of the hopper before it was filled to a depth of $37 \mathrm{~cm}$. The walls of the hopper were then raised. As the bottom of the walls rose above the boards, the boards fell free suddenly increasing the size of the hopper opening to the width of the boards. For the unweighted tests, boards narrower than $W_{\text {minn,meas }}$ determined without boards did not consistently increase of decrease $W_{\text {min,meas. }}$. For the weighted tests, adding boards narrower than $W_{\text {min,meas }}$ determined without boards increased $W_{\text {min,meas }}$. Adding boards wider than $W_{\text {min,meas }}$ resulted in flow as soon as the boards fell free. 

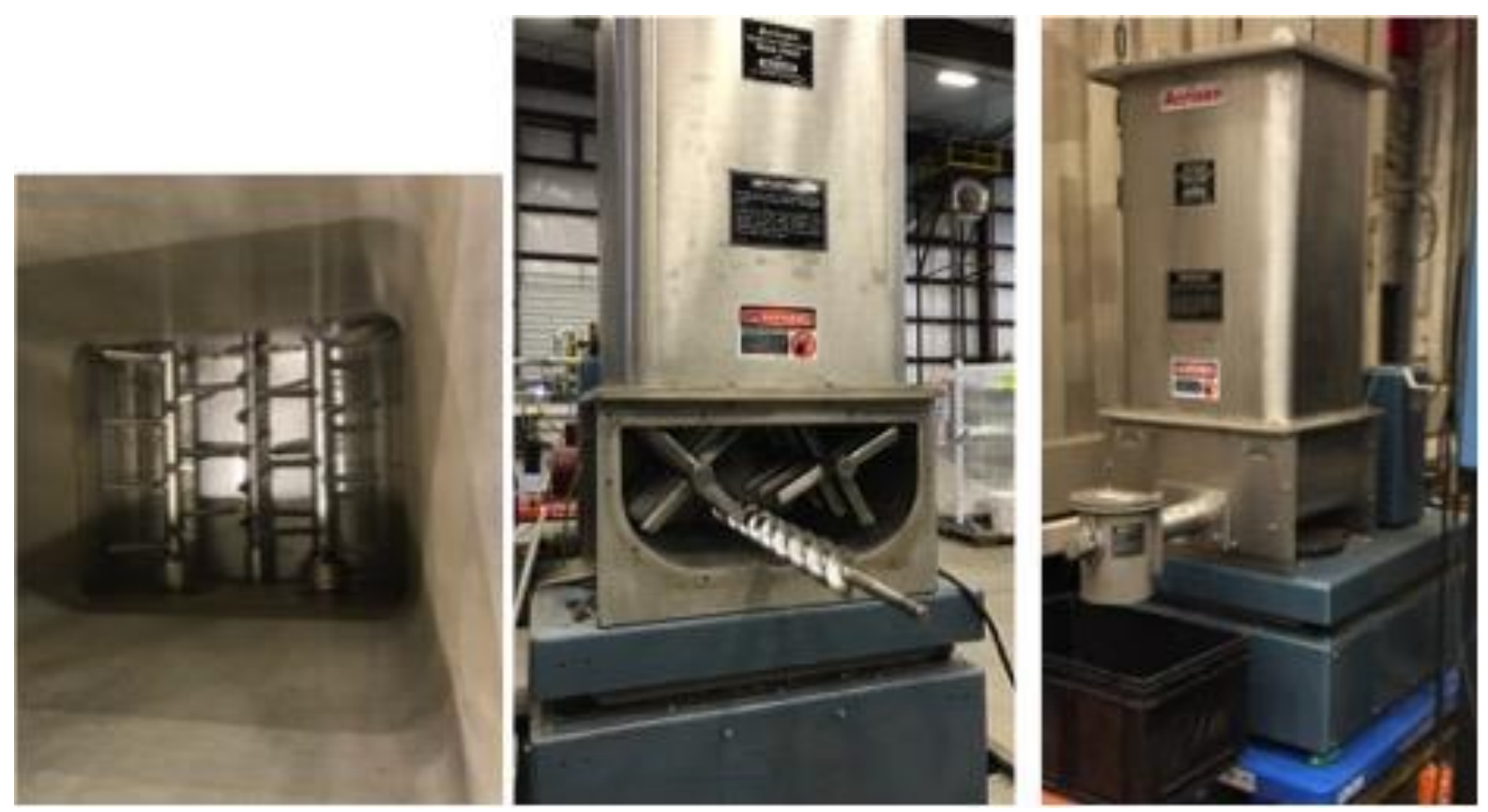

Figure A.6. Photographs of the Acrison Bin Weight Screw Feeder (model 402X-250-75BDF1.5-E/2). The left image is a top view of the bin auger feeder with a screw auger and two conditioning agitators on either side. The middle image shows the bin with the front panel removed, and the right image shows the assembled bin with the auger housing intact. 

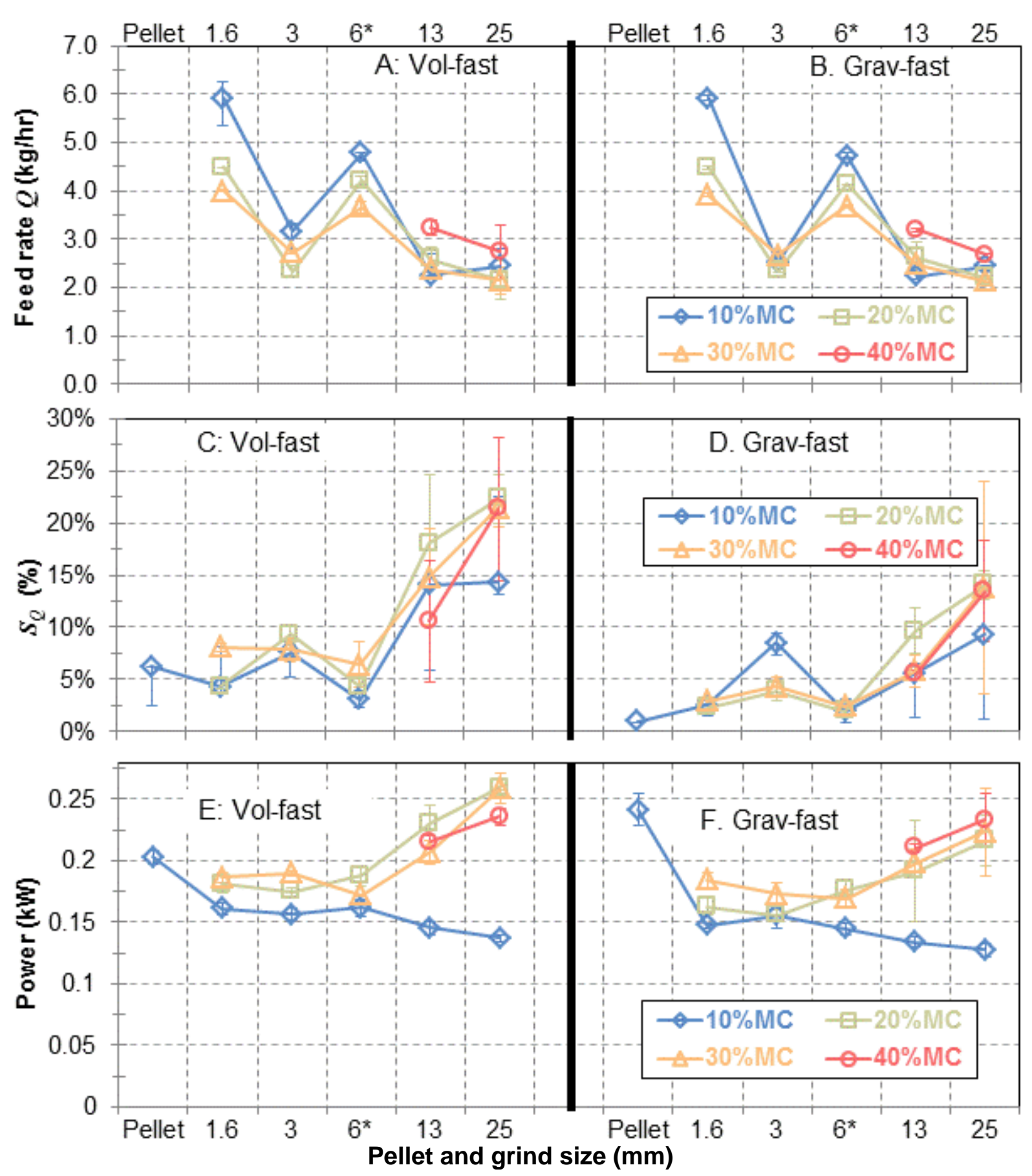

Figure A.7. Measured feed rates (Panels A and B), time variation of the feed rate $S_{Q}$ (Panels C and D) and power consumption (Panels $\mathrm{E}$ and $\mathrm{F}$ ) for experiments that employed the small auger at fast feed rate. Panels A, C, and E show results for the volumetric-mode tests, whiles panels B, $\mathrm{D}$, and $\mathrm{F}$ show corresponding results from the gravimetric-mode tests. The Vol-fast and Gravfast feed rate of the pellets were 21.3 and $28.6 \mathrm{~kg} / \mathrm{hr}$, respectively (not shown). Uncertainty bars indicate the difference between repeated tests and were 0.55 and $0.05 \mathrm{~kg} / \mathrm{hr}$ for the pellet feed rates in Vol-fast and Grav-fast operating modes, respectively (not shown). 

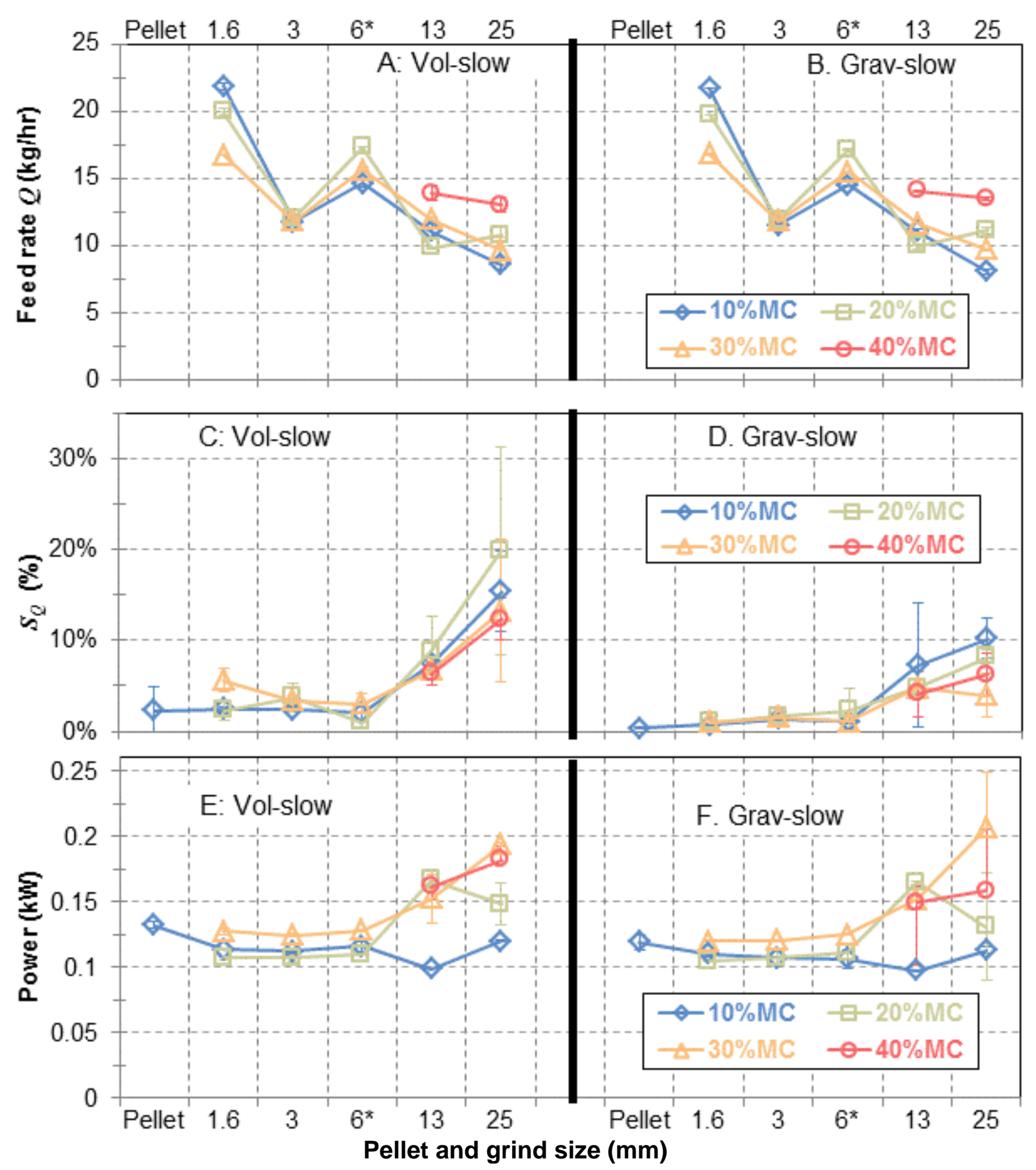

Figure A.8. Measured feed rates (Panels A and B), time variation of the feed rate $S_{Q}$ (Panels C and D) and power consumption (Panels $\mathrm{E}$ and $\mathrm{F}$ ) for experiments that employed the large auger at slow feed rate. Panels A, C, and E show results for the volumetric-mode tests, whiles panels B, $\mathrm{D}$, and $\mathrm{F}$ show corresponding results from the gravimetric-mode tests. The Vol-slow and Gravslow feed rate of the pellets were 69.7 and $64.2 \mathrm{~kg} / \mathrm{hr}$, respectively (not shown). Uncertainty bars indicate the difference between repeated tests and were 1.3 and $0.27 \mathrm{~kg} / \mathrm{hr}$ for the pellet feed rates in Vol-slow and Grav-slow operating modes, respectively (not shown). 

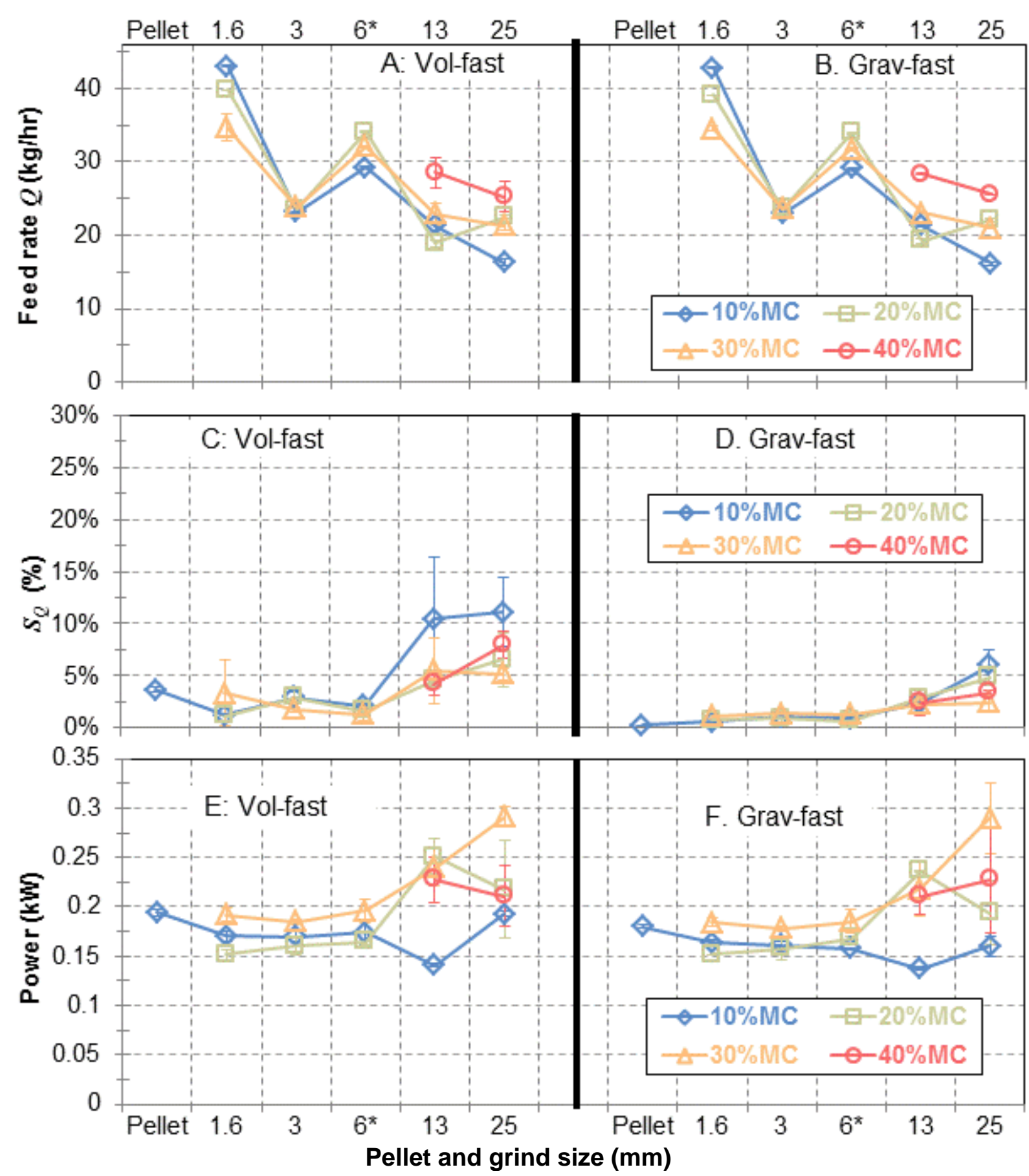

Figure A.9. Measured feed rates (Panels A and B), time variation of the feed rate $S_{Q}$ (Panels C and D) and power consumption (Panels $\mathrm{E}$ and $\mathrm{F}$ ) for experiments that employed the large auger at fast feed rate. Panels A, C, and E show results for the volumetric-mode tests, whiles panels B, $\mathrm{D}$, and $\mathrm{F}$ show corresponding results from the gravimetric-mode tests. The Vol-fast and Gravfast feed rate of the pellets were 135.1 and $136.0 \mathrm{~kg} / \mathrm{hr}$, respectively (not shown). Uncertainty bars indicate the difference between repeated tests and were 0.13 and $0.05 \mathrm{~kg} / \mathrm{hr}$ for the pellet feed rates in Vol-fast and Grav-fast operating modes, respectively (not shown). 


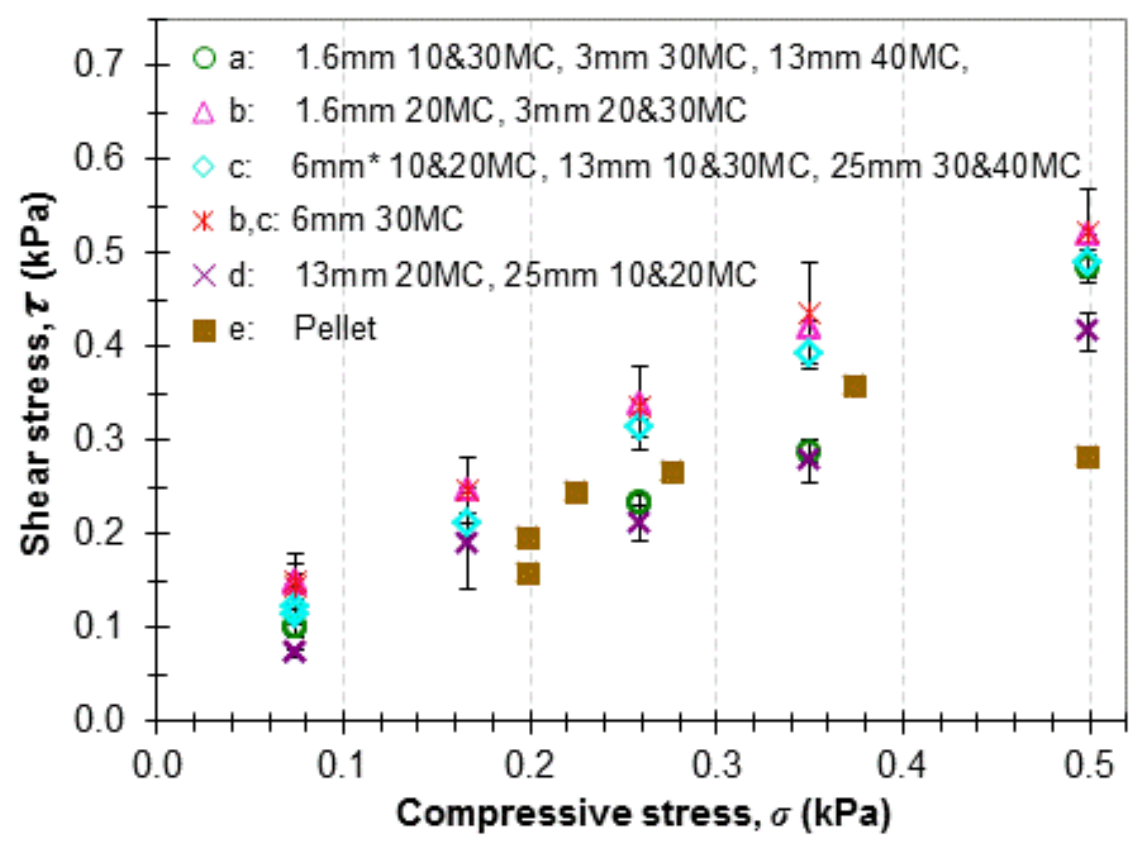

Figure A.10. Grouped instantaneous yield loci (IYL) of the woody samples for a preshear compression stress of $0.5 \mathrm{kPa}$. Groups were formed by combining samples according to the set designations in Appendix A Table A.1. Uncertainty bars show the standard deviation of all measurements within each group. Note: shear tests using pellets with a preshear stress of $0.5 \mathrm{kPa}$ did not produce reliable or useful IYLs. For the purposes of predicting hopper performance, additional shear tests for pellets were performed using a preshear stress of $1.0 \mathrm{kPa}$ (See Fig. A.12).

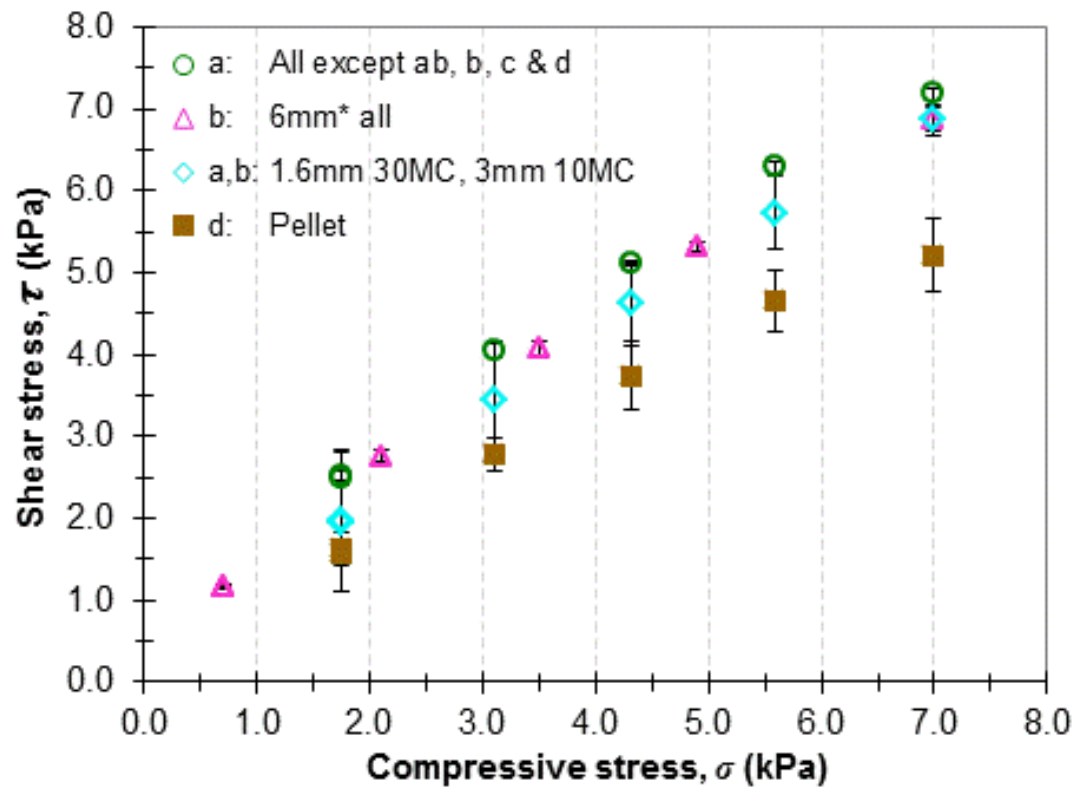

Figure A.11. Grouped instantaneous yield loci (IYL) of the woody samples for a preshear compression stress of $7.0 \mathrm{kPa}$. Groups were formed by combining samples according to the set designations in Appendix A Table A.1. 


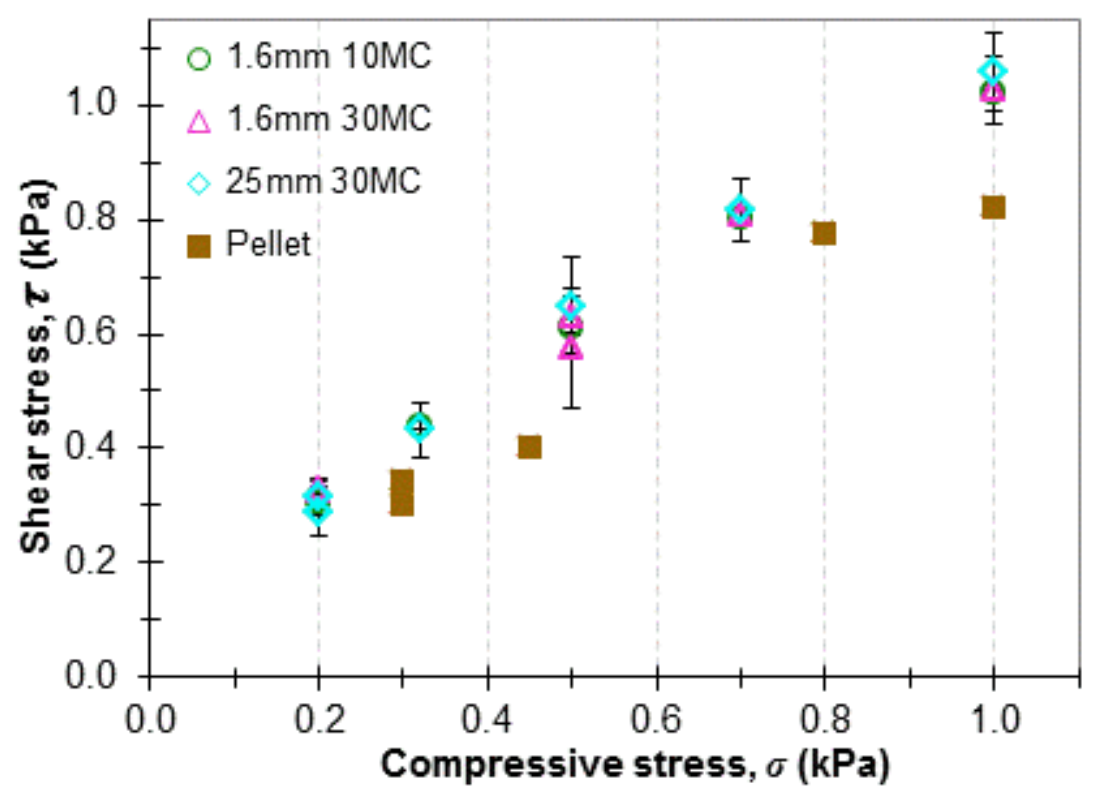

Figure A.12. Grouped instantaneous yield loci (IYL) of the woody samples for a preshear compression stress of $1.0 \mathrm{kPa}$. Each material was tested three times. For the pellets, two of the tests failed.

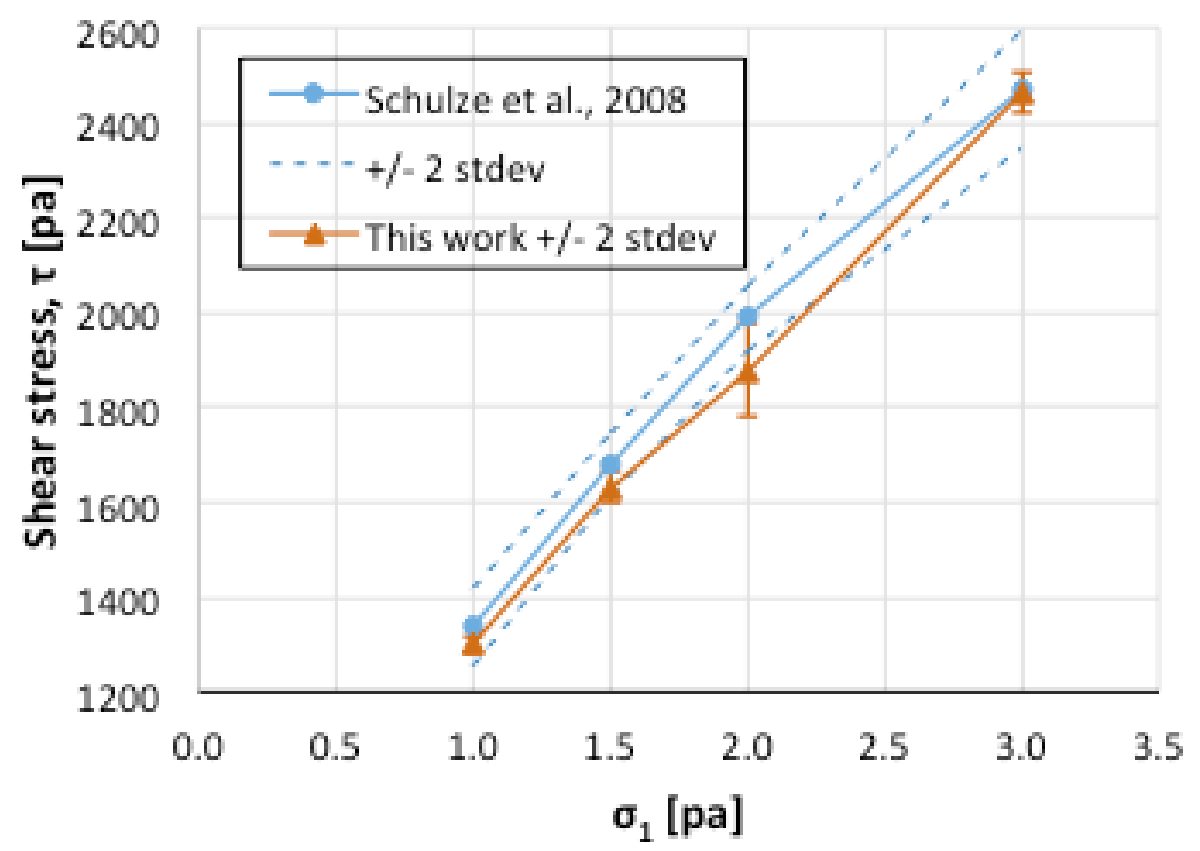

Figure A.13. Instantaneous yield loci (IYL) of BCR® certified Reference Material (limestone, Signma Aldrich, 207-439-9) for a preshear compression stress of $3.0 \mathrm{kPa}$. Three replicates were performed. Results from a 27 lab round-robin using limestone powder CRM-116 are shown for comparison. The results presented here are slightly lower than those from the round-robin, [D. Schulze, Round robin test on ring shear testers, Adv. Powd. Technol. (2011) 197-202], but are 
within or close to 2 standard deviations. The difference between this and the past results is likely due to small differences in the materials, which came from different suppliers several years apart.

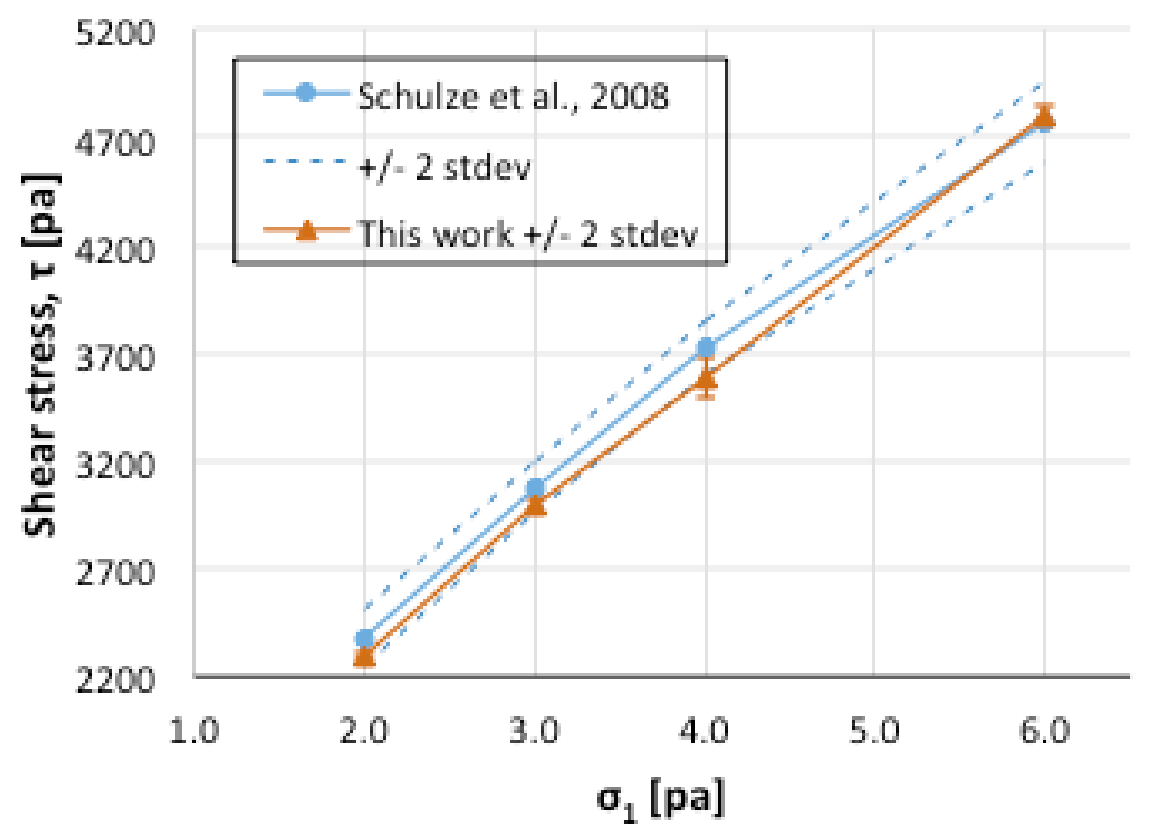

Figure A.14. Instantaneous yield loci (IYL) of BCR® certified Reference Material (limestone, Signma Aldrich, 207-439-9) for a preshear compression stress of $6.0 \mathrm{kPa}$. Three replicates were performed. Results from a 27 lab round-robin using limestone powder CRM-116 are shown for comparison.

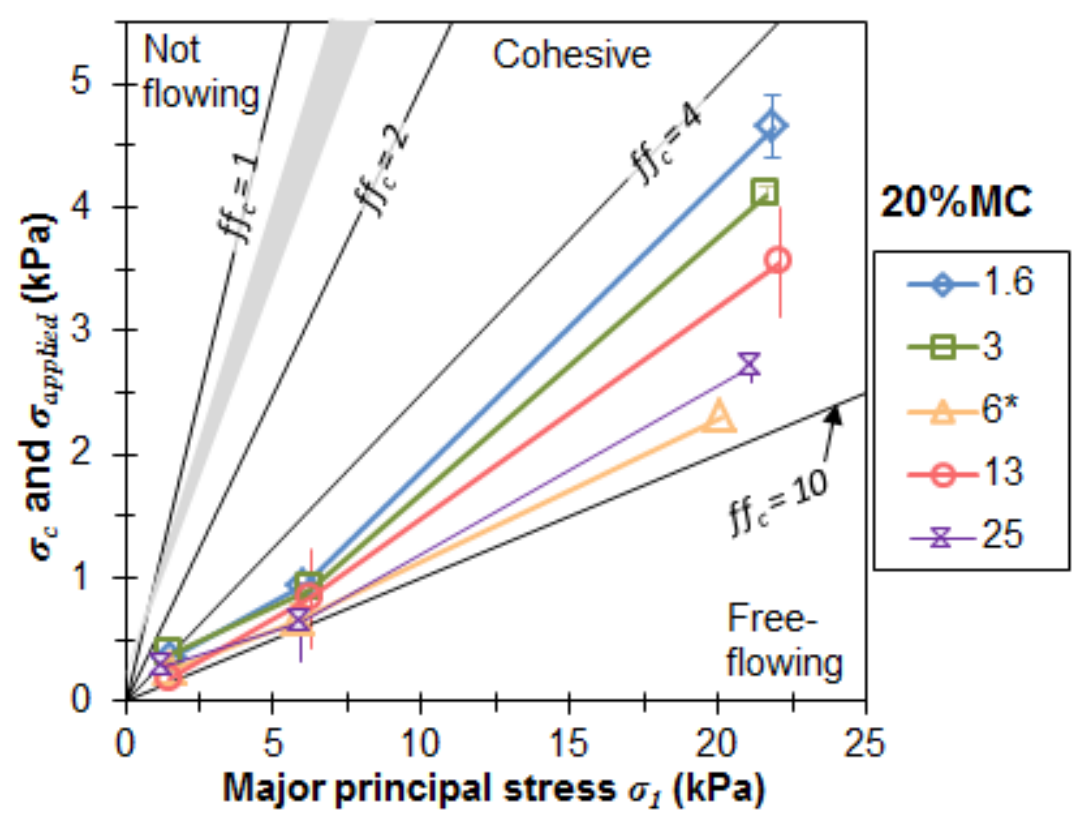


Figure A.15. Flow functions of the $20 \% \mathrm{MC}$ samples. The range of inverse hopper flow factors $(1 / f f)$ is shown as a gray wedge, and lines of constant flowability, $f f$, are also shown for comparison.

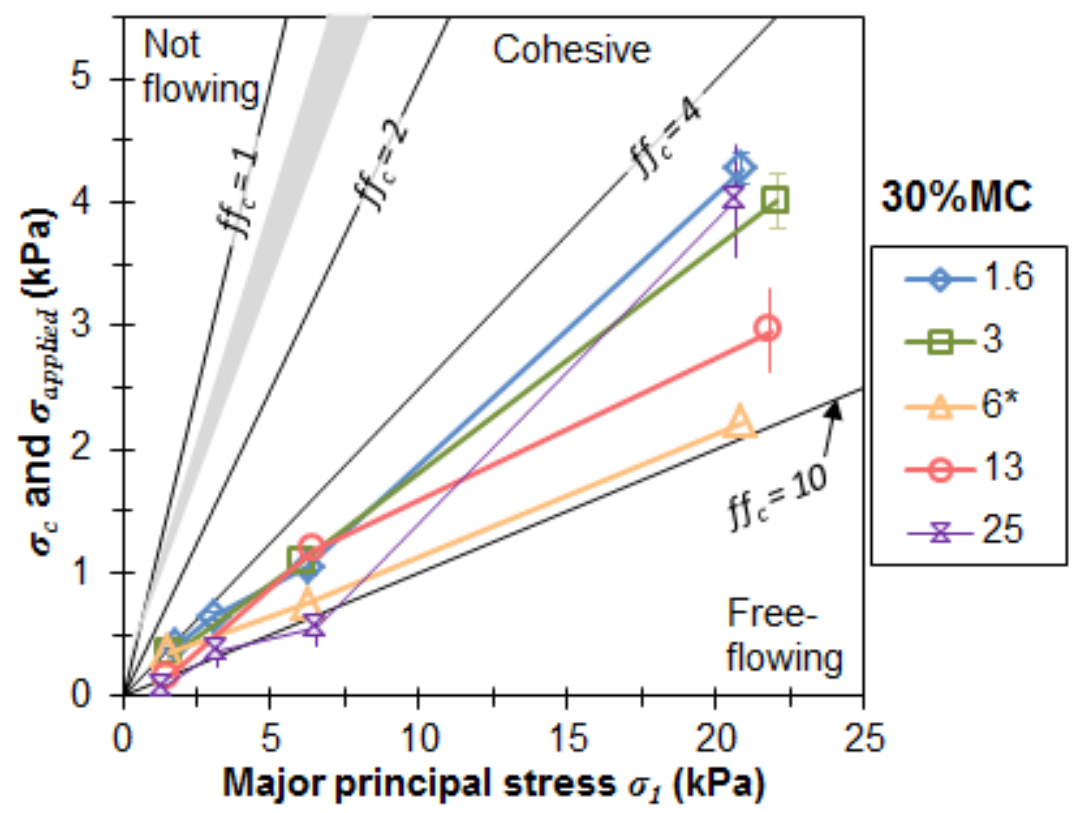

Figure A.16. Flow functions of the $30 \% \mathrm{MC}$ samples. The range of inverse hopper flow factors $(1 / f f)$ is shown as a gray wedge, and lines of constant flowability, $f f c$, are also shown for comparison.

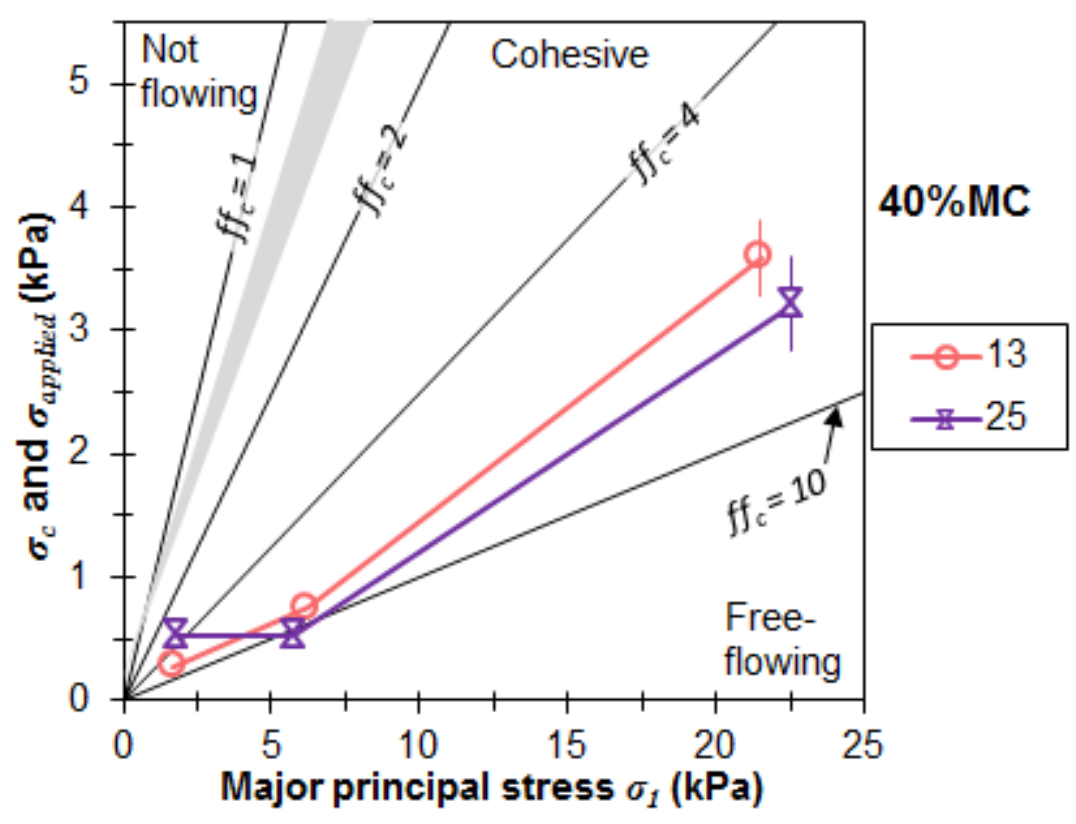


Figure A.17. Flow functions of the $40 \% \mathrm{MC}$ samples. The range of inverse hopper flow factors $(1 / f f)$ is shown as a gray wedge, and lines of constant flowability, $f f c$, are also shown for comparison.

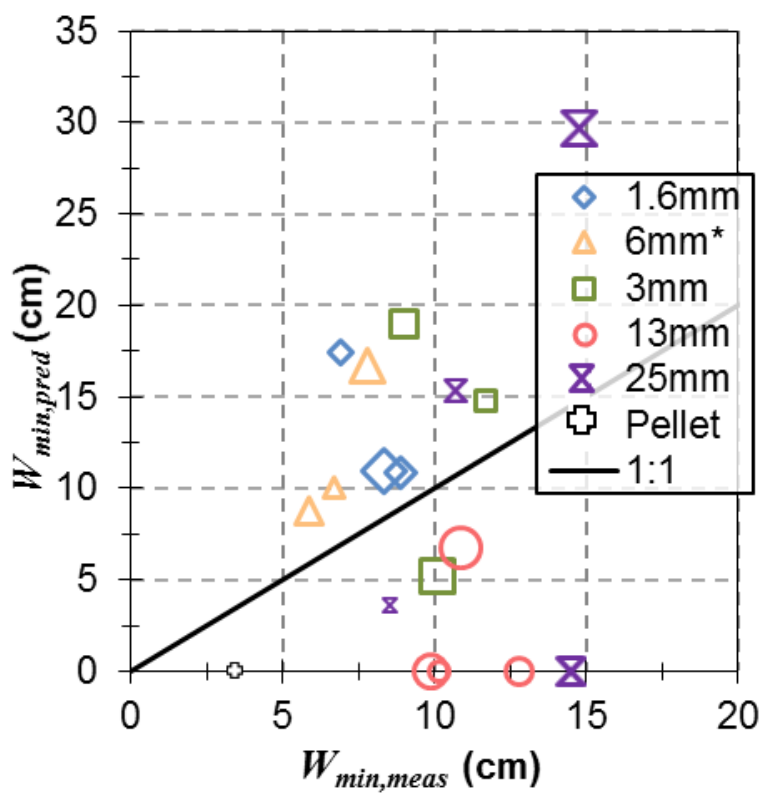

Figure A.18 Predicted minimum hopper outlet widths using the hydrostatic approach versus the values measured using the hopper tests. Symbol size indicates moisture content with larger symbols representing higher moisture content. Predicitons are based upon material properties measured using a preshear stress of $2.0 \mathrm{kPa}$.
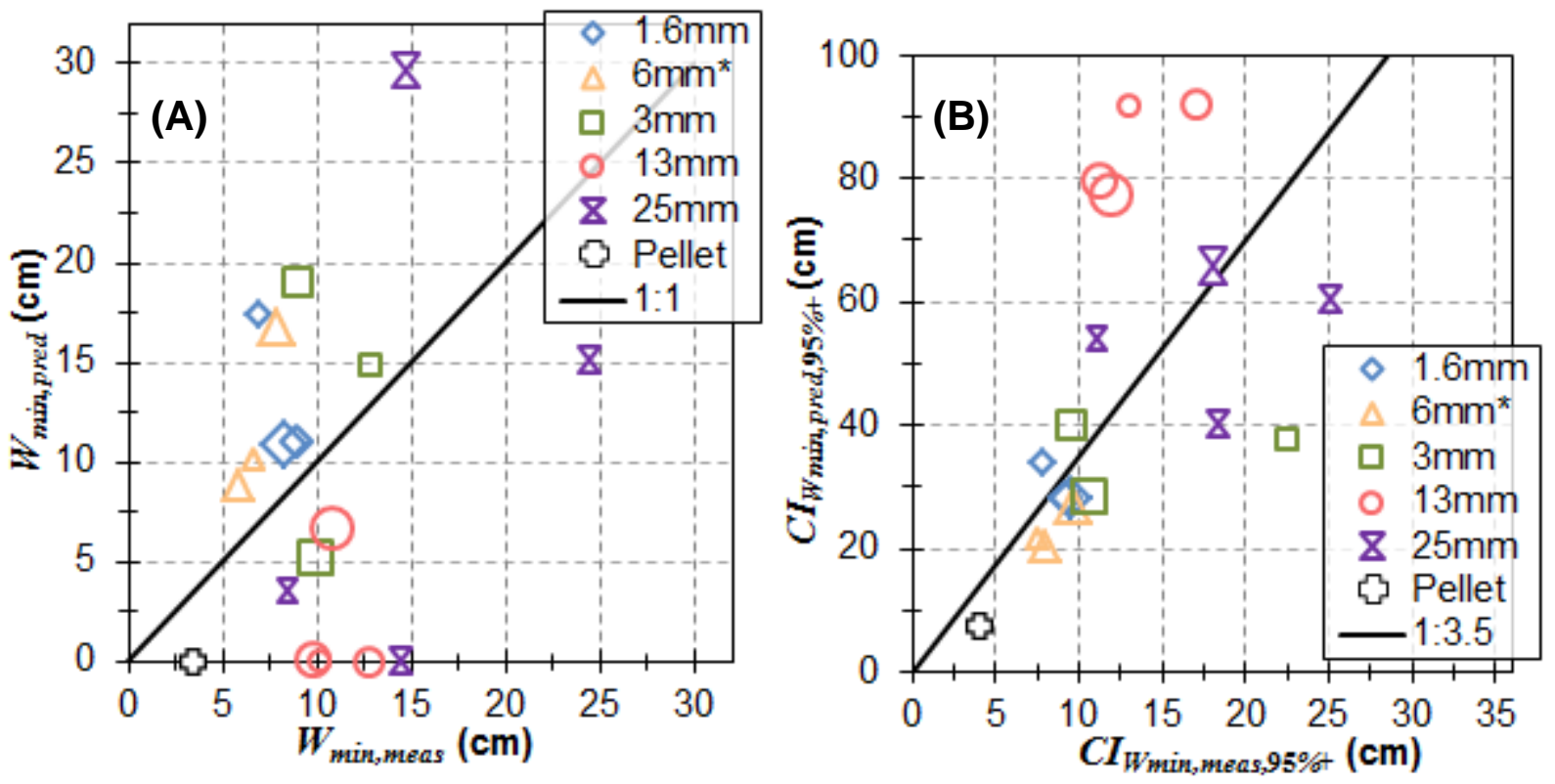
Figure A.19. Predicted minimum hopper outlet widths versus the values measured using the hopper tests. Symbol size indicates moisture content with larger symbols representing higher moisture content. Predicitons are based upon material properties measured using a preshear stress of $2.0 \mathrm{kPa}$.
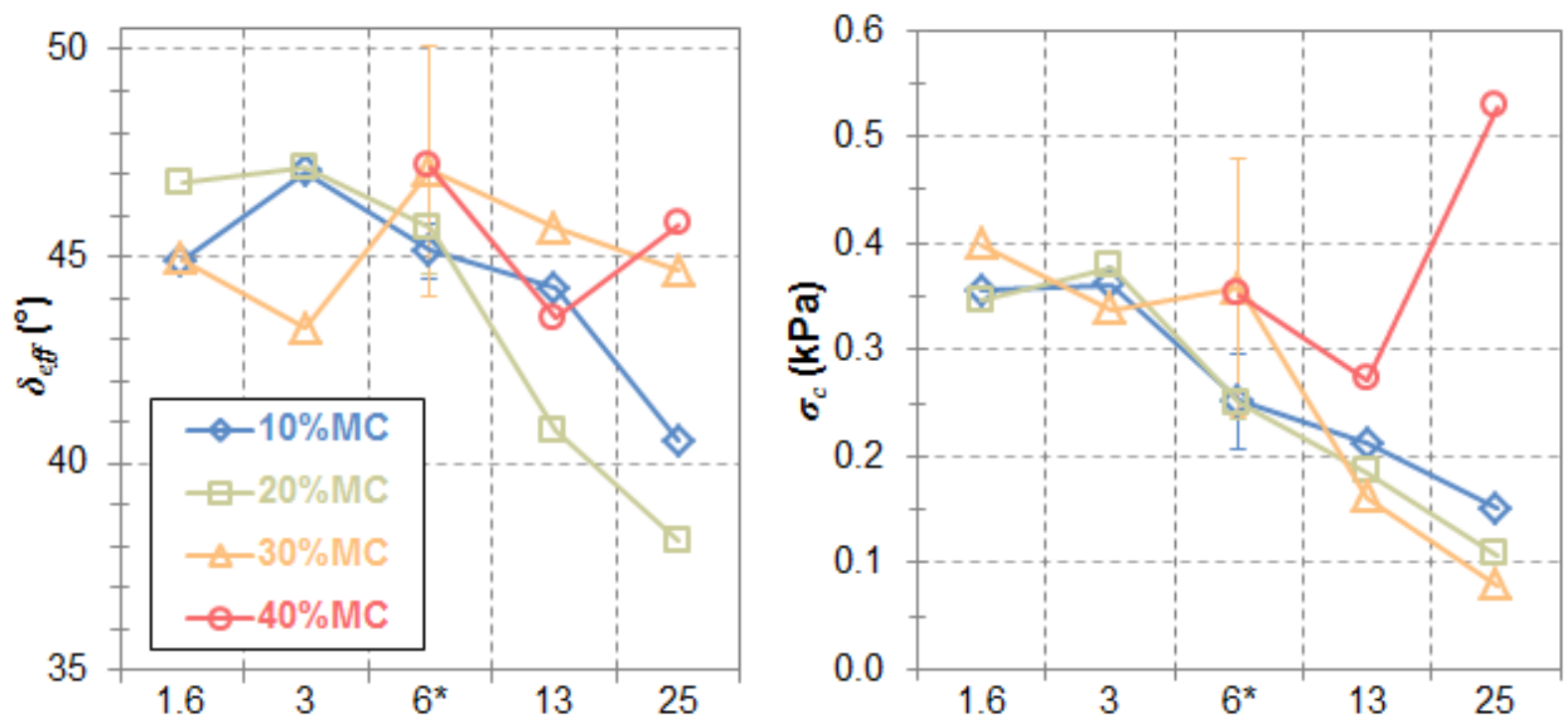

Figure A.20. Effective angle of internal friction $\delta_{\text {eff }}$ (panel A) and unconfined yield strength $f_{c}$ (panel B) for pine samples as measured using an automated Schulze ring shear tester. The preshear stress condition was $0.5 \mathrm{kPa}$ for all tests.
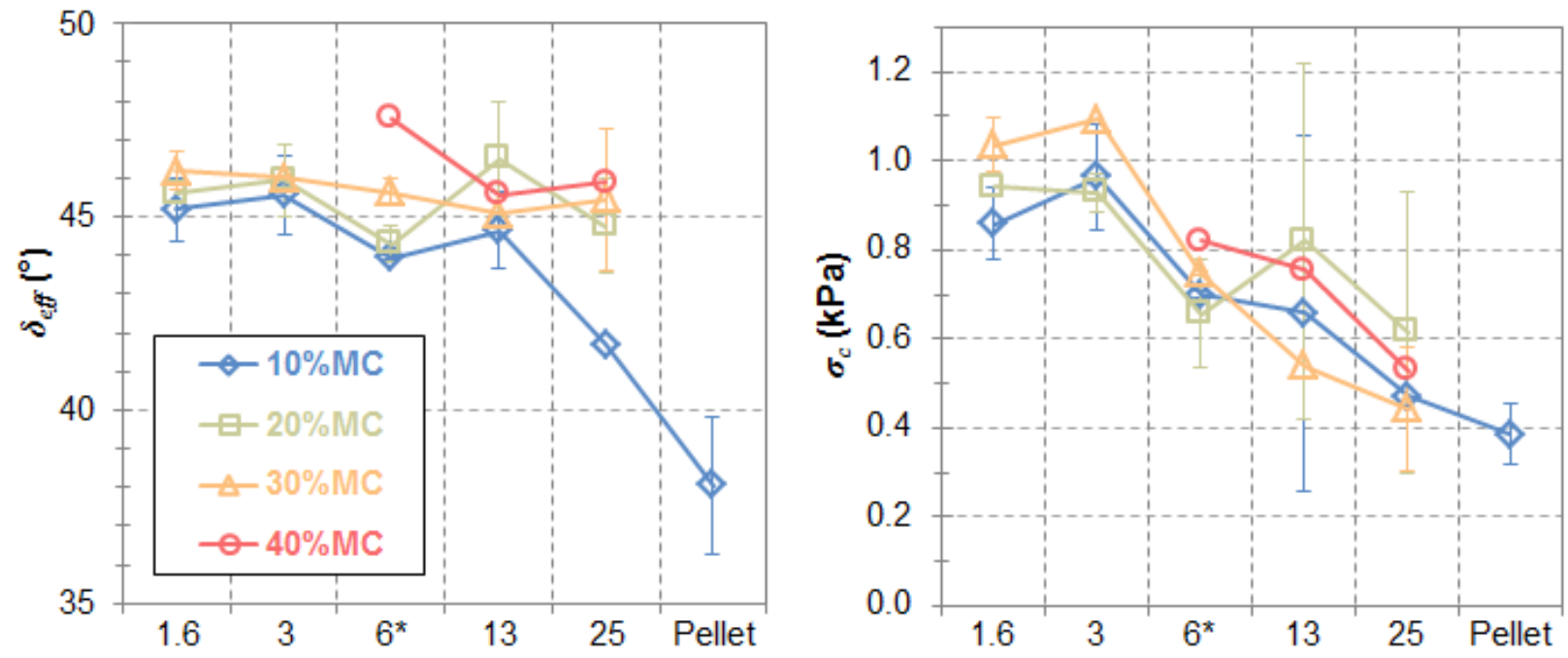

Figure A.21. Effective angle of internal friction $\delta_{\text {eff }}$ (panel A) and unconfined yield strength $f_{c}$ (panel B) for pine samples as measured using an automated Schulze ring shear tester. The preshear stress condition was $2.0 \mathrm{kPa}$ for all tests. 

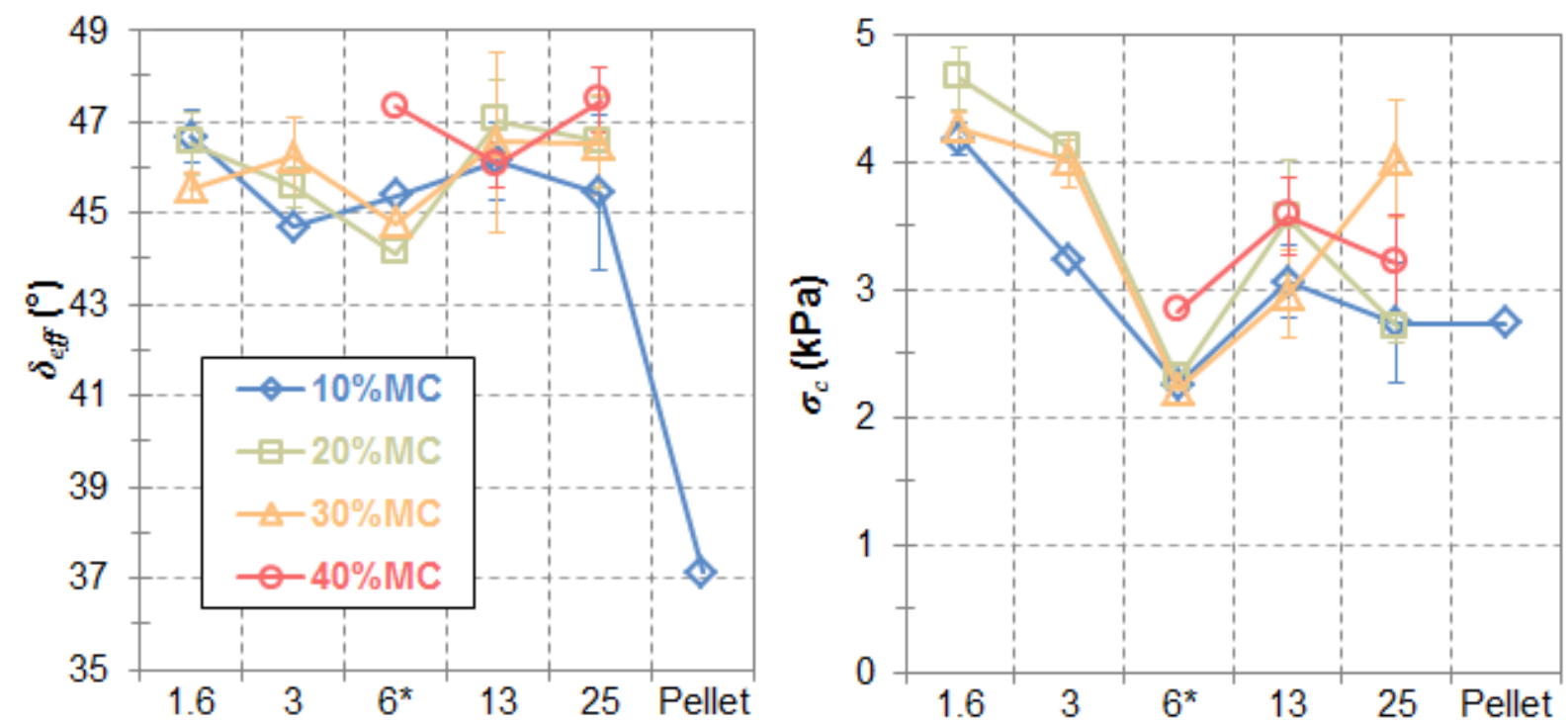

Figure A.22. Effective angle of internal friction $\delta_{\text {eff }}$ (panel A) and unconfined yield strength $f_{c}$ (panel B) for pine samples as measured using an automated Schulze ring shear tester. The preshear stress condition was $7.0 \mathrm{kPa}$ for all tests.

Figures A.20 and A.21 show that $\sigma_{c}$ (also labeled $f_{c}$ ) tends to decrease as the grind size increases above $3 \mathrm{~mm}$ (i.e. the material becomes weaker and more flowable at free surfaces). $\delta_{\text {eff }}$ does not show a strong dependence upon grind size or moisture content, except for the $25 \mathrm{~mm}$ grind at $10 \%$ MC, which is lower than the other samples. Similar trends for $\theta_{\text {eff }}$ are observed for preshear stresses of $0.5 \mathrm{kPa}$ and $7 \mathrm{kPa}$, except that at $7 \mathrm{kPa}$, the 13 and $25 \mathrm{~mm}$ grinds retain strength that is comparable to that of the finer grinds.

The $25 \mathrm{~mm}$ ground material with $10 \%$ and $20 \%$ MCs contents appear to deviate from the trend established by the other materials, as the same materials did in the shear tests that measured the

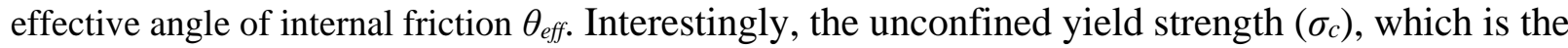
parameter most often used to predict arching in hoppers and other equipment, does not follow the same trend. 